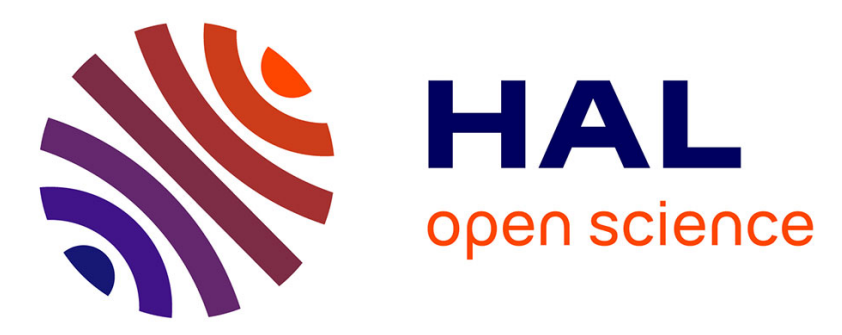

\title{
Numerical modeling of 3D zero-offset laboratory data by a discretized Kirchhoff integral method
}

Anastasiya Tantsereva, Bjørn Ursin, Nathalie Favretto-Cristini, Paul Cristini, Arkady Aizenberg

\section{> To cite this version:}

Anastasiya Tantsereva, Bjørn Ursin, Nathalie Favretto-Cristini, Paul Cristini, Arkady Aizenberg. Numerical modeling of 3D zero-offset laboratory data by a discretized Kirchhoff integral method. Geophysics, 2014, 79 (2), pp.T77-T90. 10.1190/geo2013-0034.1 . hal-00977806

\section{HAL Id: hal-00977806 https://hal.science/hal-00977806}

Submitted on 28 Apr 2016

HAL is a multi-disciplinary open access archive for the deposit and dissemination of scientific research documents, whether they are published or not. The documents may come from teaching and research institutions in France or abroad, or from public or private research centers.
L'archive ouverte pluridisciplinaire HAL, est destinée au dépôt et à la diffusion de documents scientifiques de niveau recherche, publiés ou non, émanant des établissements d'enseignement et de recherche français ou étrangers, des laboratoires publics ou privés. 


\title{
Numerical modeling of 3D zero-offset laboratory data by a discretized Kirchhoff integral method
}

\author{
Anastasiya Tantsereva ${ }^{1}$, Bjørn Ursin ${ }^{1}$, Nathalie Favretto-Cristini ${ }^{2}$, \\ Paul Cristini ${ }^{2}$, and Arkady M. Aizenberg ${ }^{3}$
}

\begin{abstract}
Accurate simulation of seismic wave propagation in complex geologic structures is of particular interest nowadays. However, difficulties arise for complex geologic structures with great and rapid structural changes, due, for instance, to the presence of shadow zones, head waves, diffractions and/ or edge effects. Different methods have thus been developed and are typically tested on synthetic configurations against analytical solutions for simple canonical problems, reference methods, or via direct comparison with real data acquired in situ. Such approaches have limitations, especially if the propagation occurs in a complex environment with strong-contrast reflectors and surface irregularities because it can be difficult to determine the method that gives the best approximation of the "real" solution or to interpret the results obtained without an a priori knowledge of the geologic environment. An alternative approach for seismics consists in comparing the
\end{abstract}

synthetic data with data obtained in laboratory experiments. In contrast to in situ experiments, high-quality data are collected under controlled conditions for a known configuration. In contrast with numerical experiments, laboratory data possess many of the characteristics of field data because real waves propagate through models with no numerical approximations. Our main purpose was to test the approach of using laboratory data as reference data for benchmarking 3D numerical methods and techniques using the setup that we have designed for this study. We performed laboratory-scaled measurements of zero-offset reflection of broadband pulses from a strong topographic environment immersed in a water tank. We compared these measurements with numerical data simulated by means of a discretized Kirchhoff integral method. The comparisons of synthetic and laboratory data indicated a good quantitative fit in terms of time arrivals and acceptable fit in amplitudes. Thus, the first step of the approach was successfully applied.

\section{INTRODUCTION}

Accurate simulation of seismic wave propagation in complex geologicl structures is of particular interest nowadays due to its applicability in various forward and inverse problems (e.g., Ursin, 2004; Robertsson et al., 2007; Virieux and Operto, 2009; Virieux et al., 2011). It is widely used for environmental and industrial applications for subsurface structure evaluation and in various aspects of seismic exploration, such as seismic data acquisition, data processing, interpretation, and as a core tool of seismic imaging and inversion methods.
In models with simple structures and slowly varying material properties, conventional methods (e.g., ray methods and finite-difference methods) are efficient. However, difficulties arise for complex geologic structures with great and rapid structural changes, and conventional methods may then fail to simulate realistic wavefields due to the presence of shadow zones, head waves, diffractions and/ or edge effects. Numerous attempts have been made to improve seismic modeling methods for complex geologic models with structural complexities such as faults with steep dips or curved reflectors.

Different methods have thus been developed and are typically tested on synthetic configurations against analytical solutions for

\footnotetext{
Manuscript received by the Editor 29 January 2013; revised manuscript received 4 November 2013; published online 26 February 2014.

${ }^{1}$ Norwegian University of Science and Technology, Department of Petroleum Engineering and Applied Geophysics, Trondheim, Norway. E-mail: anastasiya .tantsereva@ntnu.no; bjorn.ursin@ntnu.no.

${ }^{2}$ Aix-Marseille University, LMA, CNRS, UPR 7051, Centrale Marseille, Marseille, France. E-mail: favretto@1ma.cnrs-mrs.fr; cristini@1ma.cnrs-mrs.fr

${ }^{3}$ Institute of Petroleum Geology and Geophysics, SB RAS, Novosibirsk, Russia. E-mail: aizenbergam@ipgg.sbras.ru.

(C) 2014 Society of Exploration Geophysicists. All rights reserved.
} 
simple canonical problems or reference methods. Several projects focusing on verification and validation of numerical methods have been conducted in the last few years. The most recent are, for example, the SPICE code validation project (Moczo et al., 2006) and its continuation, the SISMOWINE verification project, aimed at creating a long-term basis for benchmarking numerical methods and codes for seismic wave propagation and earthquake motion simulation and the SEAM project (Fehler and Keliher, 2011) used to generate synthetic data sets for benchmarking and testing new processing algorithms. Different numerical codes were compared during the latter project to choose the most suitable one. Such an approach has limitations, especially if the propagation occurs in a complex environment with strong-contrast reflectors and surface irregularities because it can be difficult to determine the method that gives the best approximation of the real solution given by a reference method. Another approach is to validate these methods via direct comparison with real data acquired in situ (Houbiers et al., 2012; Zhang et al., 2012). Unfortunately, without a priori (good) knowledge of the geologic environment, the interpretation of the obtained results may be a tedious task due to the existence of diffraction and sideswipe events.

An alternative approach for seismic simulation consists in comparing the synthetic data with data obtained in the laboratory. The main assumption underlying this approach is that scaled physical mechanisms are identical to field physical mechanisms (Ebrom and McDonald, 1994). Such an approach holds in our case because we will resort to a linear wave equation. In contrast to in situ experiments, high-quality data are collected under controlled conditions for a known configuration, which is important for comparisons with numerical propagation models. Moreover, unlike synthetic data, laboratory data possess many of the characteristics of field data (random and signal-generated noise, multiples, mode conversions), as real waves propagate through models with no numerical approximations.

Since the first part of the 20th-century small-scaled physical modeling approaches have been extensively exploited in seismic simulation for a better understanding of wave propagation phenomena by studying the kinematics (Rieber, 1936; Howes et al., 1953; Oliver et al., 1954; Woods, 1956; Angona, 1960), and for validation of theoretical predictions by using amplitude measurements (Grannemann, 1956; Roever et al., 1959; Hilterman, 1970; Howson and Sinha, 1984; Pant et al., 1992; Chen and McMechan, 1993). The advent of computing technologies has led to application of physical model data in testing seismic processing and imaging techniques (French, 1974; Macdonald et al., 1987; Lo et al., 1988). Initially, the main reason for developing physical modeling for studying complex wave propagation was the low level of computational power that made numerical modeling unrealistic. But even nowadays, physical modeling is still frequently used for configurations whose response is difficult to model numerically. It has been used to study wave propagation in complex 3D media, such as anisotropic (Stewart et al., 2013), random heterogeneous (Sivaji et al., 2002), dynamic (Sherlock and Evans, 2001), fractured (Ekanem et al., 2013), or anelastic (Lines et al., 2012) media. Data from laboratory experiments are considered as input data in inverse problems (Pratt, 1999), for testing new data processing algorithms (Campman et al., 2005), in time-lapse 3D studies (Sherlock et al., 2000), and to benchmark numerical model solutions (Bretaudeau et al., 2011).
Our aim is to study 3D complications in zero-offset reflection profiles acquired over a strong topographic environment to improve the understanding of the physical mechanisms involved in the interaction of the waves with irregular surfaces. As noted previously, in such a complex environment the numerical methods based on approximations may fail to simulate accurately the seismic wavefields and produce different results depending on their intrinsic hypotheses. The main purpose of this article is therefore to test the approach of using laboratory data as reference data for benchmarking 3D numerical methods and techniques using the Marseille model setup that we have specially designed for this study. The Marseille model is partly based on French's model (French, 1974). In addition to a plane fault and a full dome, it contains original features such as a truncated dome and a truncated pyramid. The presence of the edges and the corners of these structures is expected to complicate the wavefields significantly. Using the indoor tank facilities of the Laboratoire de Mécanique et d'Acoustique (LMA, Marseille, France), we have performed laboratory-scaled measurements of zero-offset reflection of broadband pulses on the Marseille model immersed in a water tank. In what follows, we will present comparisons of these measurements with numerical data simulated by means of a discretized Kirchhoff integral method. The choice of the numerical method can be explained by several factors: it is computationally inexpensive and fast, still able to model piecewise smooth interfaces (at least approximately); and it allows for modeling events independently, which leads to an easier interpretation of the results. The discretized Kirchhoff integral method can be seen as a simplified version of the tip-wave superposition method software package (TWSM) (Ayzenberg, 2008) with conventional plane-wave reflection coefficients (PWRCs) used instead of the effective reflection coefficients (ERC) suggested by the authors of the method. TWSM has been tested for various synthetic configurations, such as a plane interface against exact modeling (Ayzenberg et al., 2007), French's model against the finite-difference method (Ayzenberg, 2008; Ayzenberg et al., 2008), and flexural interfaces against the generalized ray tracing method (Ayzenberg et al., 2009). The numerical tests showed very good fit with analytical solutions, numerical methods, and asymptotic theoretical estimations in terms of traveltimes and amplitudes. It has been shown that the TWSM with ERC can be effectively applied in seismic modeling of reflections and transmissions, a wide range of caustic effects, head, diffracted, and creeping waves at smooth or irregular interfaces. TWSM with PWRC is faster and efficient in terms of memory usage and produces similar results for precritical offsets, but it can become inaccurate for near- and postcritical offsets. For the zero-offset configuration with a focused-beam source presented here, the discretized Kirchhoff integral method, i.e., the TWSM with PWRC, can be used to decrease the computational costs while still preserving the accuracy.

Here also, we will be concerned only with propagation problems. Discussion of migration problems is left for further articles. Some preliminary results of the comparison between laboratory data and numerical methods used for 2D modeling of wave propagation have already been reported at conferences (e.g., Cristini et al., 2012; Tantsereva et al., 2012). Here, we present additional results for $3 \mathrm{D}$ modeling and quantitative evaluation of the comparison between experimental and numerical data.

This paper is organized as follows: In the first section, we describe the experimental setup and present the laboratory data obtained in zero-offset configurations with narrow-beam and broad-beam 
transducers. The second section is devoted to the description of the numerical modeling method. In the third section, we compare the laboratory and synthetic data and discuss the results obtained.

\section{SMALL-SCALE SEISMIC MODELING}

\section{Experiment description}

Several laboratory experiments were carried out at LMA in Marseille, France. Wave propagation occurs in small-scale conditions; i.e., for instance, if a scaling factor $\mu=2 \cdot 10^{4}$ is considered, an experimental frequency of $500 \mathrm{kHz}$ corresponds to a real frequency of $25 \mathrm{~Hz}$, and an experimental distance of $10 \mathrm{~mm}$ corresponds to a real distance of $200 \mathrm{~m}$, velocities as well as densities and attenuations remaining unchanged.

The model used in these experiments, called the "Marseille model," is partly based on French's model (French, 1974), but it contains original topographies such as a truncated dome and a truncated pyramid (see Figure 1). The model of size $600 \times 400 \times$ $70 \mathrm{~mm}$ is made of PVC, which is isotropic at ultrasonic frequencies and whose elastic properties are in the same range as those of typical geologic media. The thickness of the model is from 30 to $70 \mathrm{~mm}$, the difference between the two levels separated by a planar fault being $40 \mathrm{~mm}$. The domes are parts of the spheres on the lower level with the radii equal to 90.83 and $51.25 \mathrm{~mm}$ for the truncated and full dome, respectively, the radius of their base being equal to $100 \mathrm{~mm}$. The height of the truncated dome is $15 \mathrm{~mm}$, and the height of the full dome is $40 \mathrm{~mm}$. The base of the truncated pyramid is a square $90 \times 90 \mathrm{~mm}$, and the height of the pyramid is $30 \mathrm{~mm}$. These values have been chosen to be much greater than the wavelength in water for the considered frequency range. The model is immersed in a water tank equipped with a computer-controlled system that allows for accurate positioning of the source and receiver. The measured properties of the materials are $c=1476-1493 \mathrm{~m} / \mathrm{s}$ (depending on the water temperature) and $\rho=1000 \mathrm{~kg} / \mathrm{m}^{3}$ in the water layer, and $V_{P}=2220 \mathrm{~m} / \mathrm{s}, V_{S}=1050 \mathrm{~m} / \mathrm{s}$, and $\rho_{\mathrm{PVC}}=$ $1412 \mathrm{~kg} / \mathrm{m}^{3}$ in the PVC. Attenuation in the PVC layer is described by the quality factors $40<Q_{P}<60$ and $27<Q_{S}<31$ for $\mathrm{P}$ - and $\mathrm{S}$-waves, respectively. Attenuation in the water is negligible.

As we consider the zero-offset seismic configuration only for these experiments, we illuminate the model using a piezoelectric transducer operated as a source and a receiver. We use two types of piezoelectric transducers with a dominant frequency equals to $500 \mathrm{kHz}$. The two transducers have different beam apertures, namely narrow-beam and broad-beam apertures, which allow us to obtain two different zerooffset data sets. Diameters of the piezoelectric elements for narrow-beam and broad-beam transducers are $D=25.4 \mathrm{~mm}$ and $D=3 \mathrm{~mm}$, respectively. The width of the main lobe of the beams generated by the transducers is $8.3^{\circ}$ and $45^{\circ}$ at $-3 \mathrm{~dB}$ for the narrow-beam and the broad-beam transducers, respectively. The response of each transducer has been measured by pointing its beam toward the air-water interface. The waveform has been filtered after acquisition, to eliminate the harmonic resonances of the transducer. Filtering consists in applying a eighth-order Butterworth filter to the acquired data, which corresponds to a low-pass filter with a cut-off frequency of $1.1 \mathrm{MHz}$. The resulting filtered source signal associated to each transducer and its frequency spectrum are indicated in Figures 2 and 3. It can be noticed from the figures that each transducer covers a wide frequency range. The distance of separation between the near-field and the far-field regions is calculated using the known formula (Zemanek, 1971)

$$
d_{F F}=D^{2} /\left(4 \lambda_{\text {water }}\right)
$$

where $\lambda_{\text {water }}=3 \mathrm{~mm}$ denotes the wavelength in water. It is equal to 55 and $7.5 \mathrm{~mm}$ for the narrow- and broad-beam transducers, respectively.

We use a conventional ultrasonic pulse-echo technique to obtain the reflection data. The piezoelectric transducers are located at a distance of $105 \mathrm{~mm}( \pm 1 \mathrm{~mm})$ or $150 \mathrm{~mm}( \pm 1 \mathrm{~mm})$ above the flat part of the model surface. The shortest distance to the model surface being 65 or $110 \mathrm{~mm}$, the far-field zone condition being thus fulfilled for any position of the transducer over the Marseille model. The acquisition design is shown in Figure 4 . The area covered by the

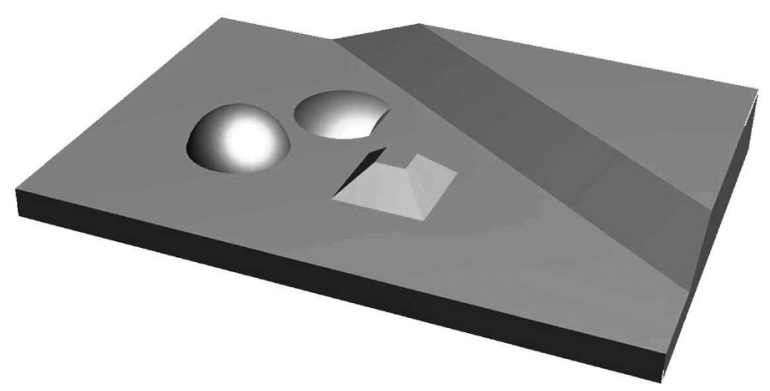

Figure 1. Marseille model with its fault, full and truncated domes, and truncated pyramid. The size of the model is $600 \times$ $400 \times 70 \mathrm{~mm}$.

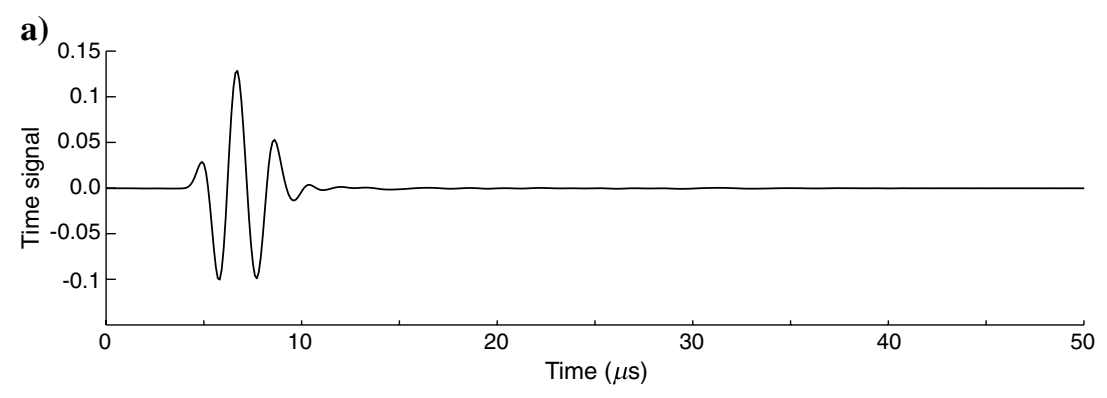

b)

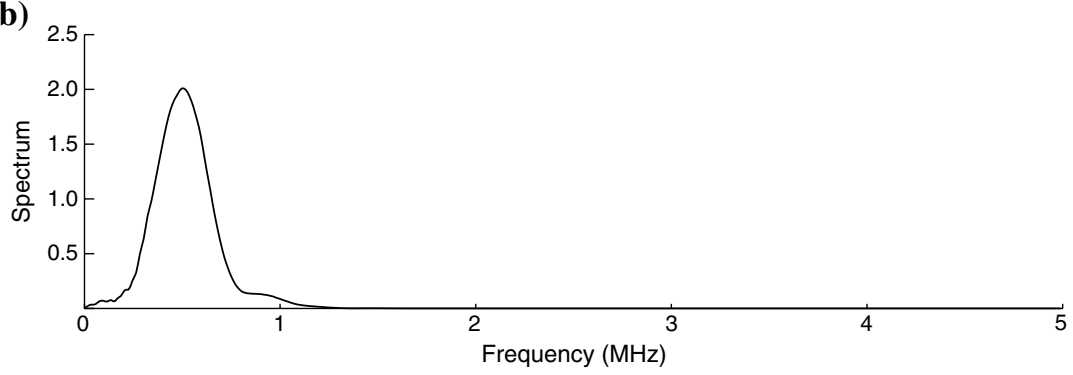

Figure 2. Time signal and associated spectrum for the narrow-beam transducer. 
acquisition is different depending on the beam width of the transducer operating as the source; i.e., it is smaller for the broad-beam transducers than for the narrow-beam ones to avoid diffractions by the edges of the model. We perform acquisitions along Y-lines with a spatial sampling $\Delta x$ equal to $2 \mathrm{~mm}$. The collected data thus consist of numerous parallel profiles composed of a collection of reflection data for dense grids of source-receiver locations. We process reflection data to produce seismograms corresponding to different cross sections of the model. We pay more attention to a few specific profiles because they are of high interest as they cross the main structures of the model. The data collected along these profiles might thus contain reflections and diffractions from all the structures.
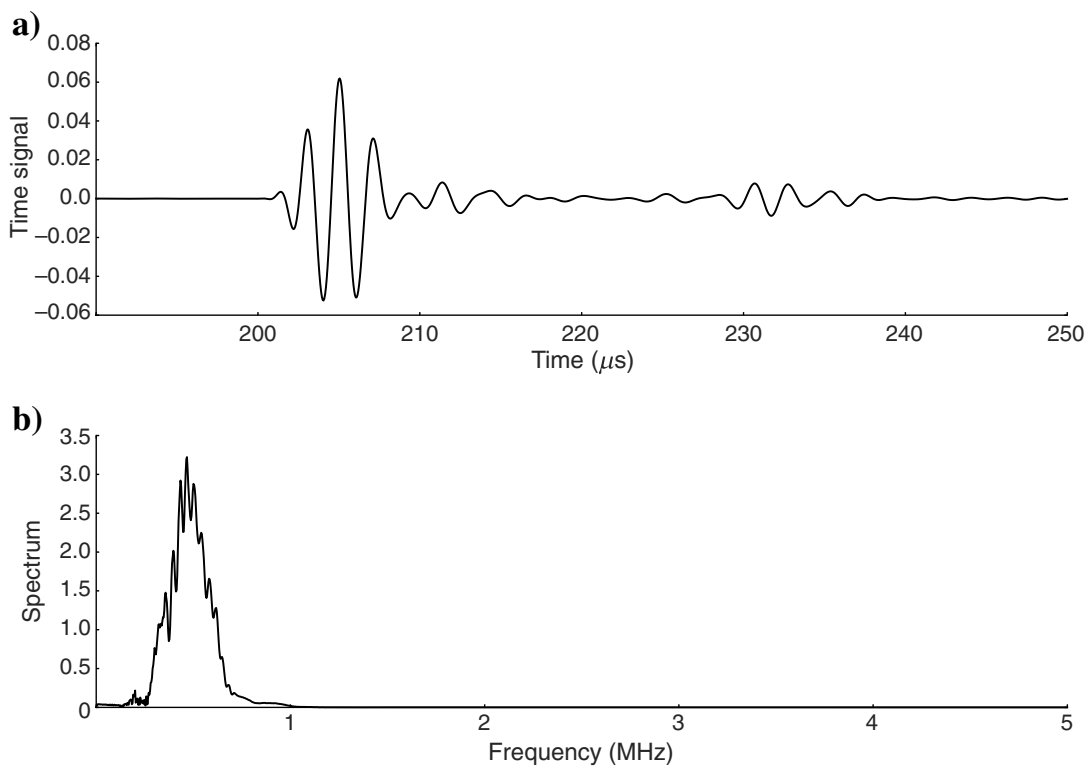

Figure 3. Time signal and associated spectrum for the broad-beam transducer.

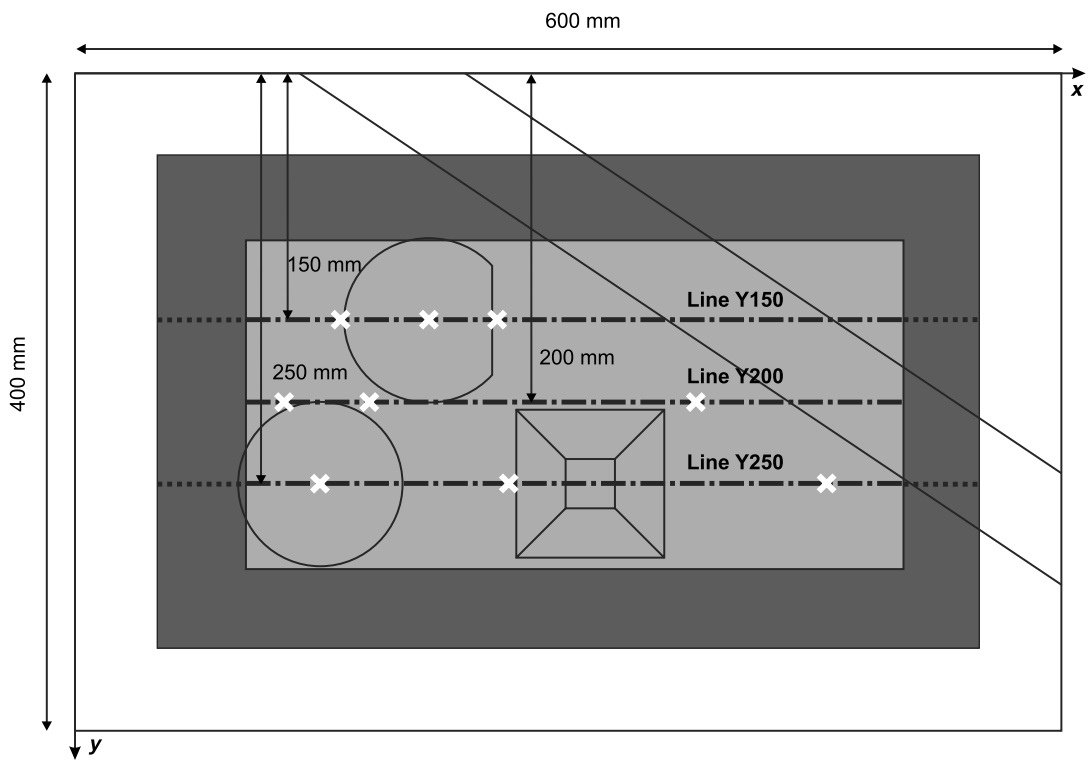

Figure 4. Acquisition design and area covered by acquisition with the narrow-beam transducer (dark gray) and with the broad-beam transducer (light gray).
These profiles are represented by dotted and dashed-dotted lines in Figure 4. Line Y150 provides the profile over the top of the truncated dome, line Y200 is located between the two domes, and line Y250 cuts the top of the full dome and the pyramid.

The seismograms are obtained after application of a low-pass filter to raw data to eliminate the harmonic resonances of the transducers. Additionally, for visualization purposes, we apply a clipping procedure to all seismograms presented below with the clipping number $x=50$, i.e., saturation of all the signals whose amplitude is greater than $x \%$ of the maximum amplitude to enlighten weaker signals. Small variations in the two-way traveltimes for reflections from the plane parts of the model can be observed. They are due to nonconstant tilt of the Marseille model during acquisition, with position errors of less than $2 \%$. Varying water temperature in the tank during long-lasting acquisitions can also have some effect on the time arrivals (see Bilaniuk and Wong [1993]) for the discussions on water velocity variations with temperature).

\section{Data for the narrow-beam source}

For the first experiment with the narrow-beam transducer, the distance from the transducer to the flat part of the model surface is $105 \mathrm{~mm}$. Figure $5 \mathrm{a}-5 \mathrm{c}$, left, shows data recorded in the laboratory along the three lines Y150, Y200, and Y250. Events with a time arrival smaller than $0.15 \mathrm{~ms}$ correspond to primary reflections from the top surface of the model. Events with a greater time arrival corresponding to either multiples or reflections from the bottom surface of the model are not shown here. One can see that the recorded signals are of high quality due to a high signal-to-noise ratio.

By qualitatively analyzing Figure $5 \mathrm{a}-5 \mathrm{c}$, we can note that the structures are partly visible only if the top of their surfaces, cut by the acquisition profiles, is quite parallel to the piezoelectric element of the transducer. For line Y200, positioned between the domes, sideswipe reflections are not visible. The steep slopes of the nontruncated dome, the pyramid, and the fault cannot be observed. Diffractions at the edges of the topographic structures also cannot be clearly observed.

\section{Data for the broad-beam source}

For the second experiment with the broadbeam transducer, the distance from the transducer to the flat part of the model surface is $150 \mathrm{~mm}$. Figure $6 \mathrm{a}-6 \mathrm{c}$, left, shows data recorded in the laboratory along the same three lines Y150, Y200, and Y250. Events with a time arrival smaller than $0.21 \mathrm{~ms}$ correspond to primary reflections from the top surface of the model. Events with a greater time arrival corresponding to either multiples or reflections from the bottom surface of the model are not shown 
here. Again, one can note that the recorded signals are of very high quality due to a high signal-to-noise ratio.

Though these data are zero-offset, they exhibit interesting 3D effects, such as diffractions at the edges of the topographic structures and reflections from the out-of-plane structures and the fault. Nevertheless, the steep slopes of the truncated pyramid remain invisible.
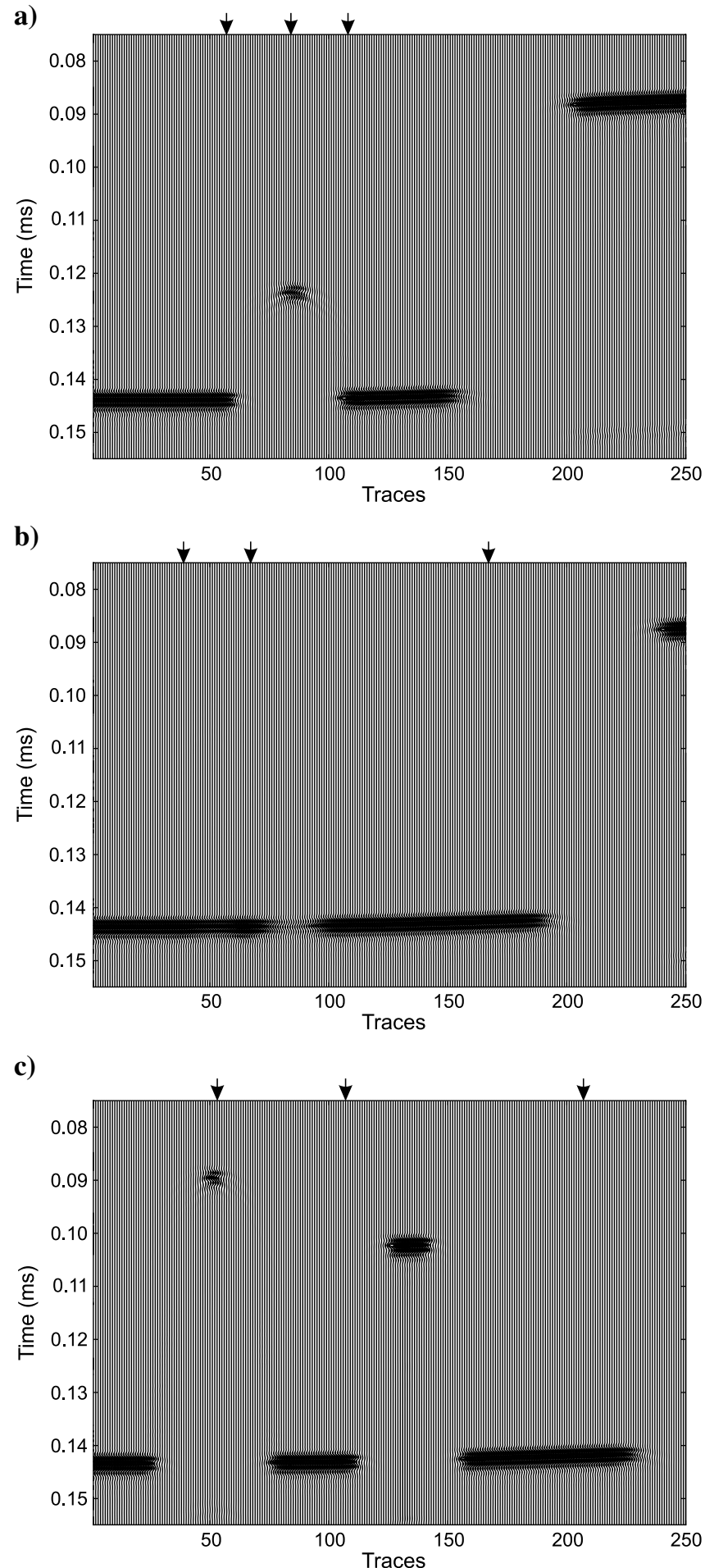

\section{NUMERICAL MODELING}

We use a discretized Kirchhoff integral method for synthetic modeling of the zero-offset experiments.

We consider the model used in the experiment described above: a homogeneous fluid medium (water) overlying an isotropic
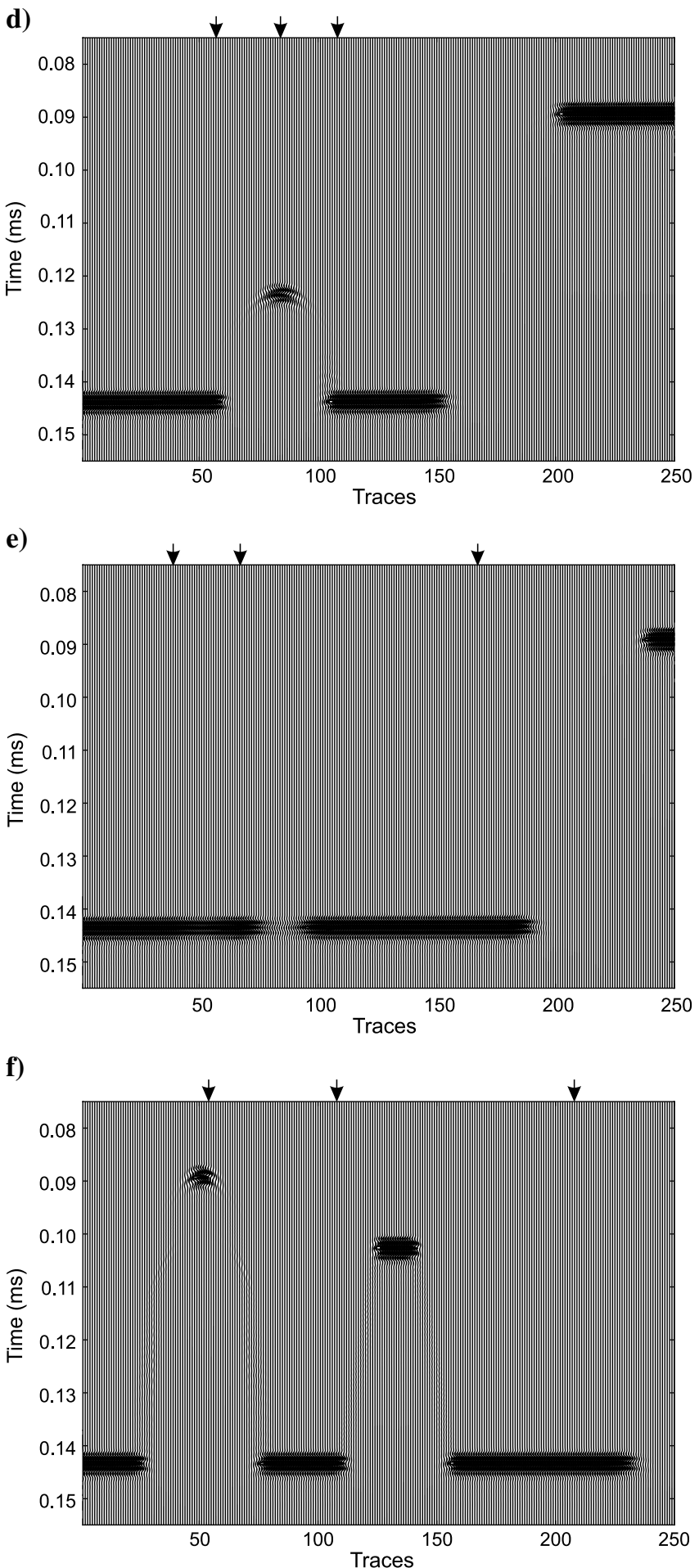

Figure 5. Laboratory seismograms (left) and single-scattering synthetic seismograms (right) obtained with the narrow-beam transducer along (a) line Y150, (b) line Y200, and (c) line Y250. 
viscoelastic medium (PVC). The fluid medium $\mathbb{D}_{1}$ is characterized by the wave velocity $c$ and the mass density $\rho$. The viscoelastic medium $\mathbb{D}_{2}$ is characterized by the $\mathrm{P}$-wave velocity $V_{P}$, $\mathrm{S}$-wave velocity $V_{S}$, the mass density $\rho_{\mathrm{PVC}}$, and the quality factors $Q_{P}$ and $Q_{S}$. The media are separated by a piecewise smooth interface
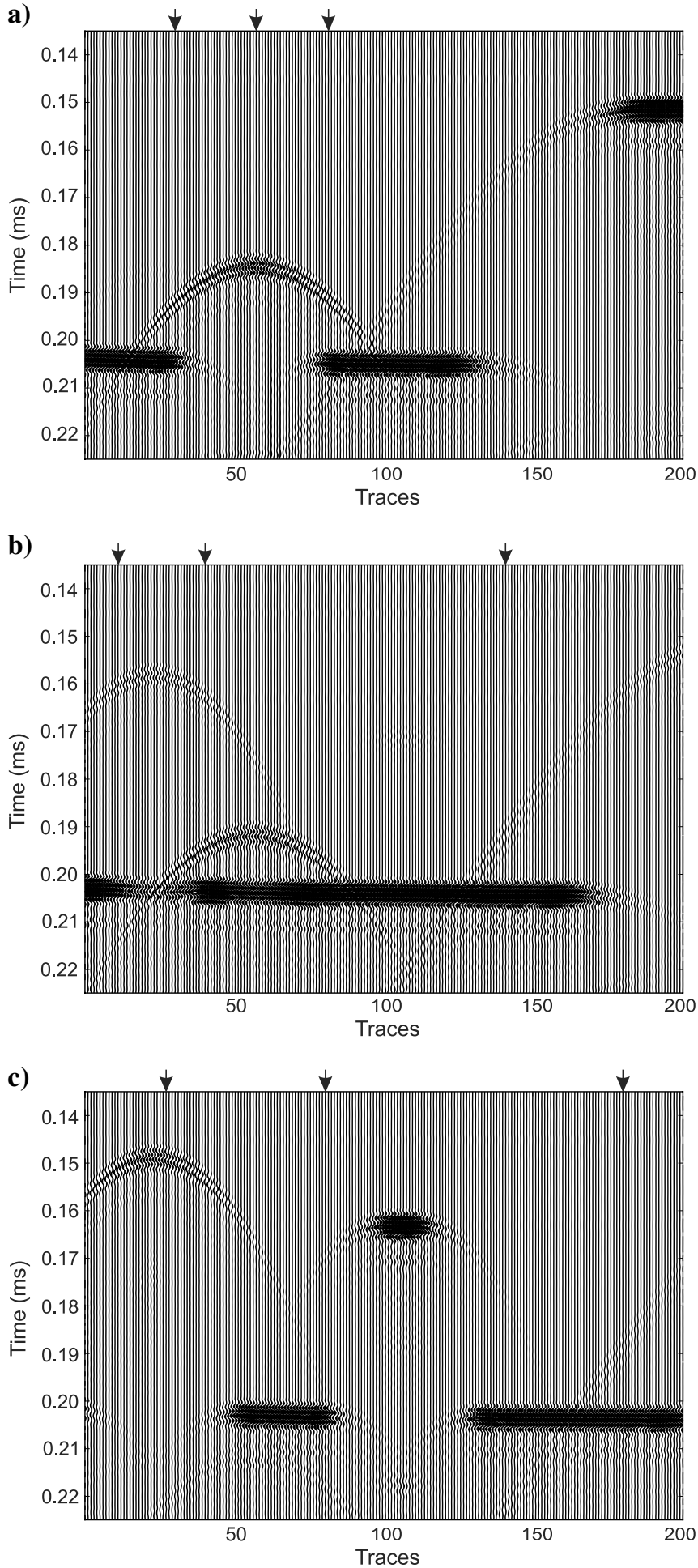

$\Sigma$. A piezoelectric disk transducer $\mathbb{S}_{D}$ of a finite diameter $D$ is immersed in the fluid and acts as a source of incident radiation and as a receiver of reflected and diffracted wavefields. We thus consider a zero-offset configuration. The vertical section of the described configuration is sketched in Figure 7.
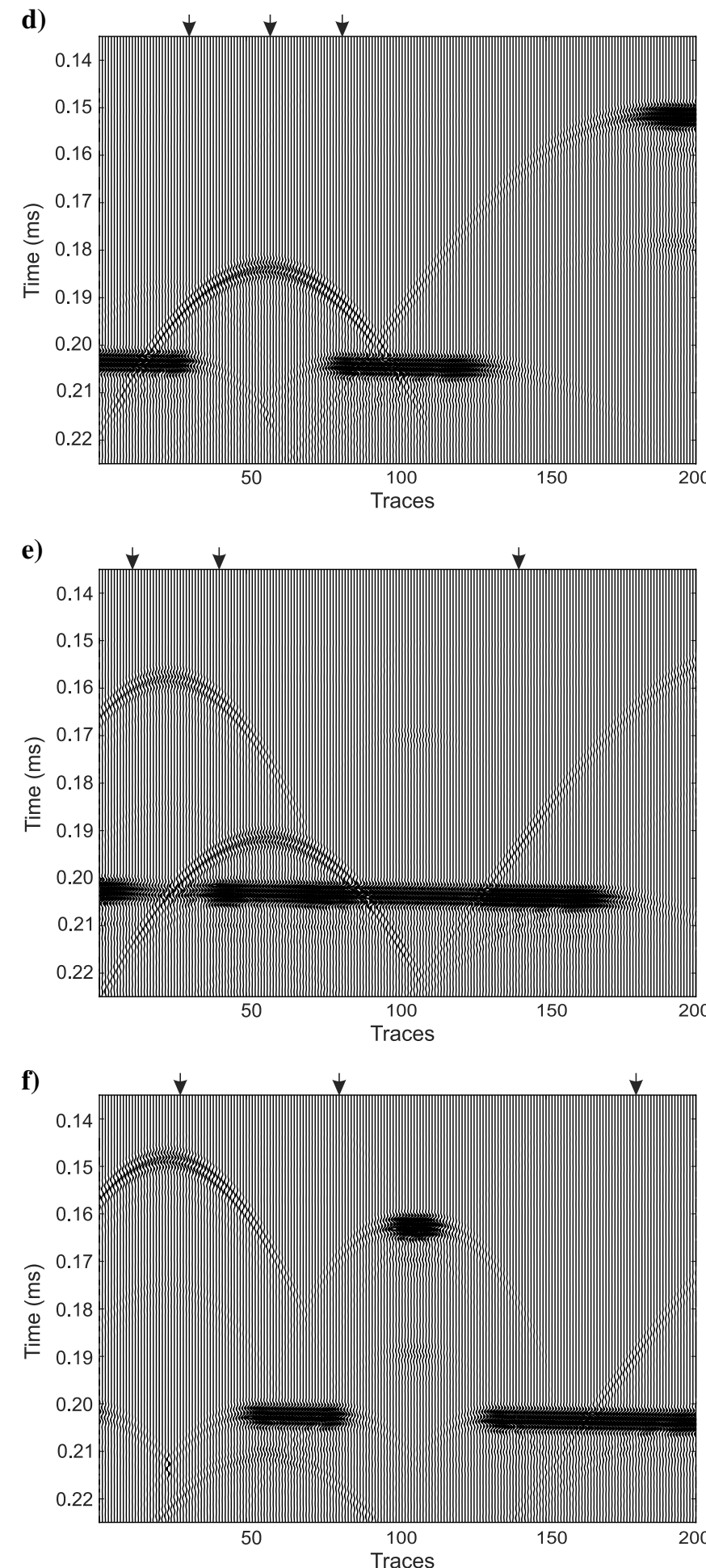

Figure 6. Laboratory seismograms (left) and single-scattering synthetic seismograms (right) obtained with the broad-beam transducer along (a) line Y150, (b) line Y200, and (c) line Y250. 
Let $\mathbf{x}=\left(x_{1}, x_{2}, x_{3}\right)$ be a fixed Cartesian coordinate system. The pressure wavefield $p(\mathbf{x}, t)$ and its spectrum $P(\mathbf{x}, \omega)$ are connected via the forward and inverse temporal Fourier transforms using the sign convention in Aki and Richards (2002) and Chapman (2004). Here, $t$ is the time and $\omega$ is the angular frequency. In the following, we shall not write the dependence on $\omega$ explicitly.

The Green's function for the Helmholtz equation

$$
\left[\nabla^{2}+\frac{\omega^{2}}{c^{2}}\right] G\left(\mathbf{x} ; x^{s}\right)=-4 \pi \delta\left(x-x^{s}\right)
$$

is

$$
G\left(\mathbf{x} ; \mathbf{x}^{s}\right)=\frac{e^{i \omega r / c}}{r},
$$

where $r=\left|\mathbf{x}-\mathbf{x}^{s}\right|$ is the distance between the points $\mathbf{x}^{s}$ and $\mathbf{x}, \mathbf{x}^{s}$ being the position of the point source.

We consider the integral representation theorem for a fluid medium bounded by a piecewise smooth interface $\Sigma$. The total wavefield from a source in the fluid consists of the direct source field, the singly scattered field, and the multiply scattered field. We shall only consider single scattering, which includes single reflections from interfaces and single diffractions from edges and vertices (Tygel and Ursin, 1999). The singly scattered pressure wavefield $P_{\mathrm{ssc}}\left(\mathbf{x}^{r}\right)$ at a given receiver point $\mathbf{x}^{r}$ can be represented by the Kirchhoff-Helmholtz surface integral (see, e.g., Haddon and Buchen, 1981):

$$
\begin{aligned}
P_{\mathrm{ssc}}\left(\mathbf{x}^{r}\right)= & \frac{1}{4 \pi} \iint_{\Sigma}\left[\frac{\partial \hat{G}\left(\mathbf{x}^{r} ; \mathbf{x}\right)}{\partial \mathbf{n}} P_{r e f}(\mathbf{x})\right. \\
& \left.-\hat{G}\left(\mathbf{x}^{r} ; \mathbf{x}\right) \frac{\partial P_{r e f}(\mathbf{x})}{\partial \mathbf{n}}\right] \mathrm{d} \Sigma(\mathbf{x}),
\end{aligned}
$$

where $\hat{G}\left(\mathbf{x}^{r} ; \mathbf{x}\right)$ is the response at the transducer at $\mathbf{x}^{r}$ from the surface point $\mathbf{x}$.

When the interface is located in the far zone with respect to the transducer, there are theoretical estimations and laboratory experiments proving that the response function $\hat{G}\left(\mathbf{x}^{r} ; \mathbf{x}\right)$ can be approximated by (see, e.g., Zemanek, 1971; Harris, 1981)

$$
\hat{G}\left(\mathbf{x}^{r} ; \mathbf{x}\right) \approx G\left(\mathbf{x}^{r} ; \mathbf{x}\right) A\left[\psi\left(\mathbf{x}^{r}, \mathbf{x}\right)\right],
$$

with the directivity function $A\left[\psi\left(\mathbf{x}^{r}, \mathbf{x}\right)\right]$, which is the same for radiation and reception, $\psi\left(\mathbf{x}^{r}, \mathbf{x}\right)$ being the angle between the normal to transducer's surface and the ray connecting points $\mathbf{x}$ and $\mathbf{x}^{r}$. For the narrow-beam transducer, it is shown in Zemanek (1971) that the directivity function is given by

$$
A\left[\psi\left(\mathbf{x}^{r}, \mathbf{x}\right)\right]=C \frac{J_{1}(\xi)}{\xi},
$$

where $\xi=\frac{\pi D}{\lambda} \sin \psi\left(\mathbf{x}^{r}, \mathbf{x}\right), J_{1}$ being the first-order Bessel function of the first kind, $C=$ const. For the broad-beam transducer, the directivity function $A\left[\psi\left(\mathbf{x}^{r}, \mathbf{x}\right)\right]$ is given by a table of discrete measured values instead of analytical representation.

Applying the Kirchhoff approximation (Bleistein, 1984), we can represent the boundary values of the reflected wavefield $P_{\text {ref }}(\mathbf{x})$ and its normal derivative at the interface $\Sigma$ in the Kirchhoff-Helmholtz integral 3 in terms of the PWRC $R\left[\theta\left(\mathbf{x}, \mathbf{x}^{s}\right)\right]$ as

$$
\begin{gathered}
P_{\text {ref }}(\mathbf{x}) \approx F(\omega) R\left[\theta\left(\mathbf{x}, \mathbf{x}^{s}\right)\right] G\left(\mathbf{x} ; \mathbf{x}^{s}\right) A\left[\psi\left(\mathbf{x}, \mathbf{x}^{s}\right)\right], \\
\frac{\partial P_{\text {ref }}(\mathbf{x})}{\partial \mathbf{n}} \approx-F(\omega) R\left[\theta\left(\mathbf{x}, \mathbf{x}^{s}\right)\right] \frac{\partial G\left(\mathbf{x} ; \mathbf{x}^{s}\right)}{\partial \mathbf{n}} A\left[\psi\left(\mathbf{x}, \mathbf{x}^{s}\right)\right],
\end{gathered}
$$

where $\theta\left(\mathbf{x}, \mathbf{x}^{s}\right)$ is the angle of incidence at the fluid-PVC contact and the Fourier transform of the source wavelet $F(\omega)$ is included.

Substituting approximation 6 in the singly scattered pressure wavefield 3 for zero-offset configuration $\mathbf{x}^{r}=\mathbf{x}^{s}$, we obtain the following approximation for the singly scattered pressure wavefield:

$$
\begin{aligned}
P_{\mathrm{ssc}}\left(\mathbf{x}^{r}\right) \approx & \frac{F(\omega)}{2 \pi} \iint_{\Sigma} \frac{\partial G\left(\mathbf{x}^{r} ; \mathbf{x}\right)}{\partial \mathbf{n}} R\left[\theta\left(\mathbf{x}, \mathbf{x}^{r}\right)\right] G\left(\mathbf{x} ; \mathbf{x}^{r}\right) \\
& \times A^{2}\left[\psi\left(\mathbf{x}, \mathbf{x}^{r}\right)\right] \mathrm{d} \Sigma(\mathbf{x}) .
\end{aligned}
$$

Approximation 7 is valid for precritical values of the incident angle $\theta$, which is a suitable assumption for zero-offset configuration.

We calculate the Kirchhoff-type surface integral 7 by discretization of the reflector into small rhombic elements. It is shown in Ayzenberg et al. (2007) that, if an element is less than a quarter of the wavelength, then surface integral 7 becomes a sum of the integrals over a plane projection of each rhombic element to the tangent plane

$$
P_{\mathrm{ssc}}\left(\mathbf{x}^{r}\right)=\sum_{m} \bar{P}_{m}\left(\mathbf{x}^{r}\right),
$$

$$
\begin{aligned}
\Delta \bar{P}_{m}\left(\mathbf{x}^{r}\right)= & \frac{F(\omega)}{2 \pi} \iint_{\Delta \Pi_{m}} \frac{\partial G\left(\mathbf{x}^{r} ; \mathbf{x}\right)}{\partial \mathbf{n}} R\left[\theta\left(\mathbf{x}, \mathbf{x}^{r}\right)\right] G\left(\mathbf{x} ; \mathbf{x}^{r}\right) \\
& \times A^{2}\left[\psi\left(\mathbf{x}, \mathbf{x}^{r}\right)\right] \mathrm{d} \mathbf{x} .
\end{aligned}
$$

In the far-field approximation, the contribution from one element can be computed as

$$
\begin{aligned}
\Delta \bar{P}_{m}\left(\mathbf{x}^{r}\right) \approx & \frac{i \omega F(\omega)}{2 \pi c} G\left(\mathbf{x}^{r} ; \mathbf{x}_{m}\right) G\left(\mathbf{x}_{m} ; \mathbf{x}^{r}\right) R\left[\theta\left(\mathbf{x}_{m}, \mathbf{x}^{r}\right)\right] \\
& \times \cos \theta\left(\mathbf{x}^{r}, \mathbf{x}_{m}\right) A^{2}\left[\psi\left(\mathbf{x}_{m}, \mathbf{x}^{r}\right)\right] \Delta \Pi_{m} .
\end{aligned}
$$

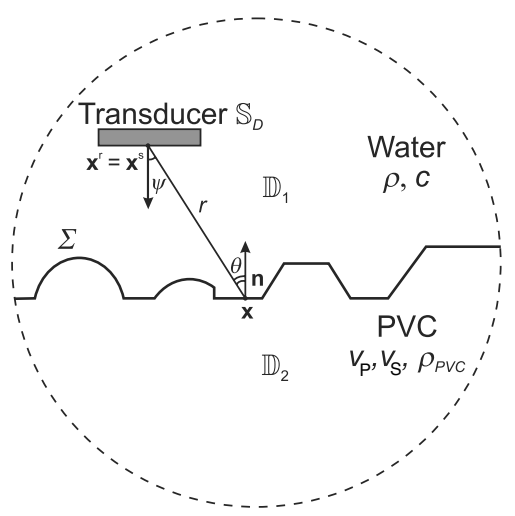

Figure 7. Vertical section of the considered model. 
We cover the model along the considered profiles with squares consisting of $200 \times 200$ square elements for 10 subsequent positions of the transducer. We choose the area of the square in such a way that it covers the surface illuminated by the transducer, taking into account the distance from the transducer to the surface and the width of the beam generated by the transducer. We choose the size of the element as a quarter of the dominant wavelength in the water to apply the above-mentioned approximations.

We use the Kolsky-Futterman model (Kolsky, 1956; Futterman, 1962) to introduce the attenuation in the PVC layer to numerical modeling. The Kolsky-Futterman model assumes that attenuation is strictly linear with the frequency over the seismic frequency range $(1-200 \mathrm{~Hz})$, which fits well with the experiments. The reflection coefficient $R\left[\theta\left(\mathbf{x}, \mathbf{x}^{r}\right)\right]$ thus becomes complex and frequencydependent as a function of complex and frequency-dependent velocities in the PVC layer.

In Figures 5 and 6, right, we show the results of modeling of the primary reflection from the top of the PVC along the Y-lines. All data sets are normalized by dividing the signal amplitude by the maximum amplitude of the whole data set acquired for the same transducer, and we apply a static time shift to the whole data set. We also apply additional trace-dependent time shifts to synthetic seismograms due to the above-mentioned data inconsistencies in traveltimes, i.e., we adjust the reflections from the plane parts of the model based on a normalized crosscorrelation coefficient.

\section{DATA COMPARISONS}

In this section, we compare the experimental data to the synthetic data. Visual observation of the total seismograms for laboratory data and single-scattering seismograms for synthetic data shows a good fit in the modeling of reflection and diffraction events (Figures 5 and 6). Let us provide a quantitative analysis by performing a numerical comparison of three selected traces for each profile line, corresponding to the source positions marked with crosses in Figure 4 and with arrows in Figures 5 and 6 . Note that the source positions are the same for narrow- and broad-beam transducers.

We use several error norms for quantitative analysis of the misfit between numerical and laboratory data. Let us consider two seismograms $s(t)$ and $s_{\text {ref }}(t)$, where $s(t)$ is the numerical signal, $s_{\text {ref }}(t)$ is the laboratory (reference) signal, and $t$ is time.

The single-valued normalized crosscorrelation coefficient $\mathrm{cc}$ is defined as (Wilks, 2011)

$$
\mathrm{cc}=\frac{\sum_{t}(s(t)-\bar{s}) \cdot\left(s_{\mathrm{ref}}(t)-\bar{s}_{\mathrm{ref}}\right)}{\sqrt{\sum_{t}(s(t)-\bar{s})^{2}} \sqrt{\sum_{t}\left(s_{\mathrm{ref}}(t)-\bar{s}_{\mathrm{ref}}\right)^{2}}},
$$

where $\bar{s}$ and $\bar{s}_{\text {ref }}$ are the mean values of the corresponding series. That crosscorrelation coefficient estimates the degree to which two signals are correlated in terms of phase and satisfies $-1 \leq \mathrm{cc} \leq 1$. The equality $\mathrm{cc}=1$ is for perfect correlation, $\mathrm{cc}=0$ for uncorrelated series, and $\mathrm{cc}=-1$ for negative correlation.

The most commonly used single-valued misfit criterion is the root mean square (rms) misfit defined as

$$
\mathrm{rms}=\sqrt{\frac{\sum_{t}\left|s(t)-s_{\mathrm{ref}}(t)\right|^{2}}{\sum_{t}\left|s_{\mathrm{ref}}(t)\right|^{2}}} .
$$

The disadvantage of using the rms misfit is that it gives the quantitative measure of the difference between the signals without indicating the reason of the difference. Moreover, it often overestimates the difference between two signals due to misinterpretation of time shifts as amplitude differences. To define the phase and amplitude misfits separately, the crosscorrelation coefficient cc can be used to determine the phase misfit (PM) and the rms misfit to determine the amplitude misfit after shifting the seismogram by the PM (see, e.g., Fehler and Keliher [2011] for more details on the technique and discussion of shortcomings of using the rms misfit alone).

However, the frequency dependence of the misfit cannot be defined this way. Therefore, in addition we consider the misfit criteria based on the time-frequency representation using the continuous wavelet transform introduced by Kristeková et al. (2009). They define a time-frequency envelope difference

$$
\Delta E(t, f)=|W(t, f)|-\left|W_{\text {ref }}(t, f)\right|
$$

and a time-frequency phase difference

$$
\Delta P(t, f)=|W(t, f)| \frac{\arg [W(t, f)]-\arg \left[W_{\text {ref }}(t, f)\right]}{\pi},
$$

where $W(t, f)$ and $W_{r e f}(t, f)$ are the complex functions of the continuous wavelet transforms of the numerical and reference signals, respectively. See Kristeková et al. (2009) for a detailed description of the time-frequency representation. The free software package TF-MISFITS can be downloaded from www.nuquake .eu/Computer_Codes/.

The time-frequency envelope misfit $(\mathrm{EM})$ is then obtained as

$$
\operatorname{TFEM}(t, f)=\frac{\Delta E(t, f)}{\max _{t, f}\left(\left|W_{r e f}(t, f)\right|\right)},
$$

and the time-frequency PM as

$$
\operatorname{TFPM}(t, f)=\frac{\Delta P(t, f)}{\max _{t, f}\left(\left|W_{r e f}(t, f)\right|\right)} .
$$

The term $\operatorname{TFEM}(t, f)$ characterizes the difference between the envelopes of the two signals as a function of time and frequency, and $\operatorname{TFPM}(t, f)$ characterizes the difference between the phases of the two signals. In addition, Kristeková et al. (2009) define time-independent (frequency envelope misfit [FEM] and frequency phase misfit $[\mathrm{FPM}]$ ) and frequency-independent (time envelope misfit [TEM] and time phase misfit [TPM]) misfits as a projection of the time-frequency misfits onto one of the two domains. Here, we will also use single-valued measures of fit based on the error norms defined above, i.e., a single-valued EM

$$
\mathrm{EM}=\sqrt{\frac{\sum_{f} \sum_{t}|\Delta E(t, f)|^{2}}{\sum_{f} \sum_{t}\left|W_{\mathrm{ref}}(f, t)\right|^{2}}}
$$




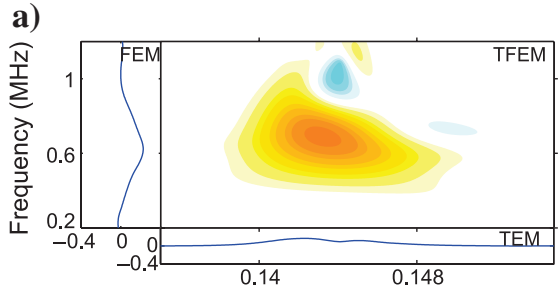

b)
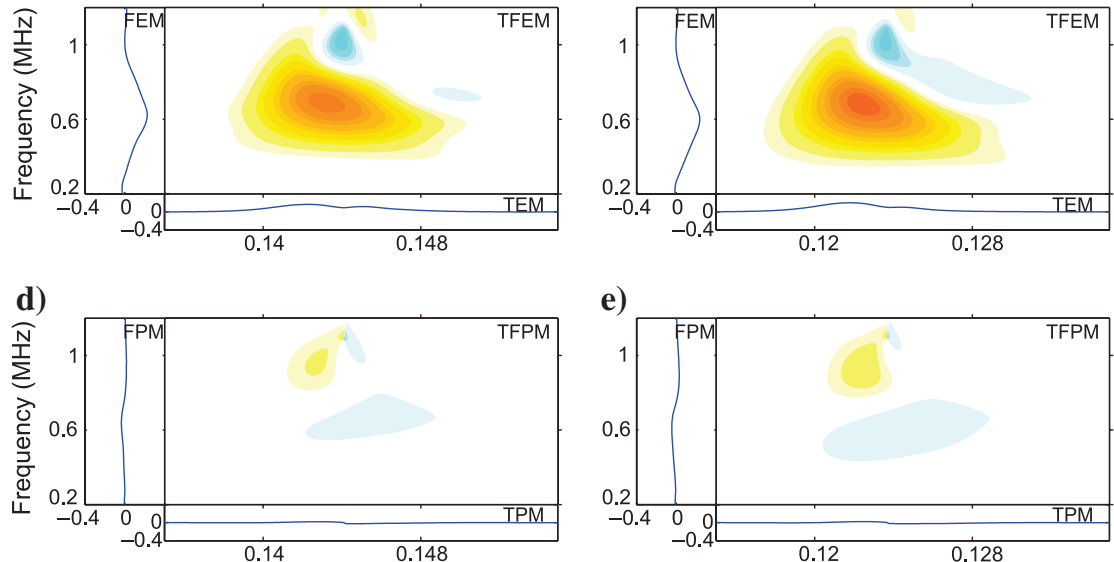

g)

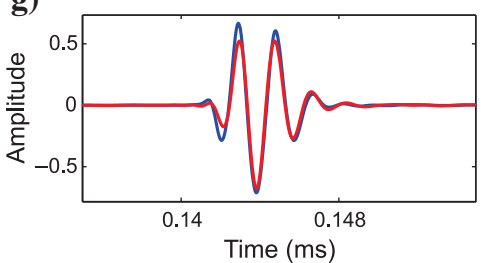

e)

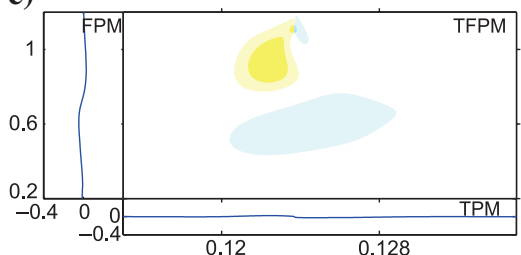

h)

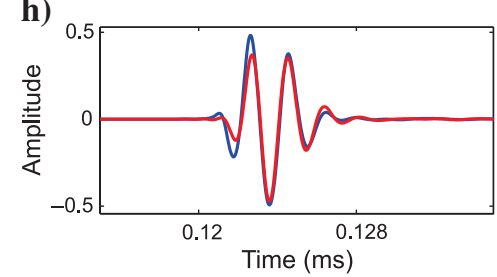

c)

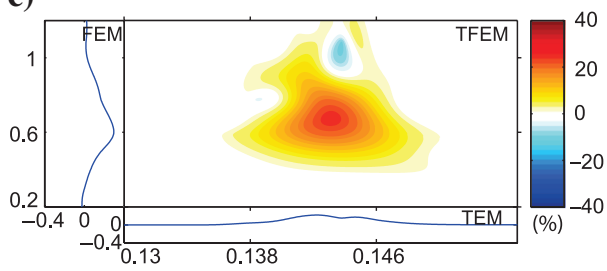

f)

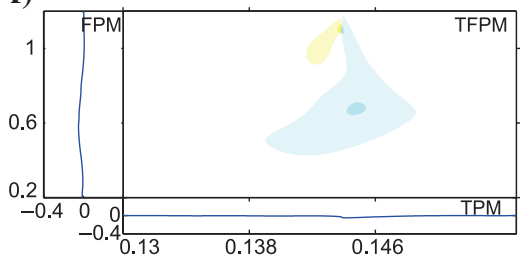

i)

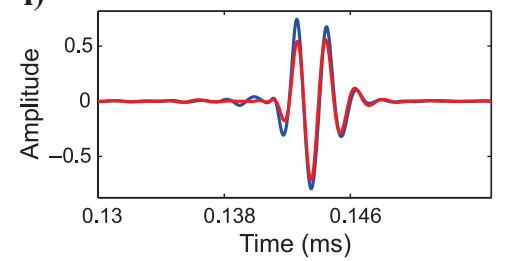

Figure 8. Time-frequency representation of the misfits for source positions NB1 (a), NB2 (b), and NB3 (c) for the narrow-beam transducer along line Y150. (a) Time-frequency envelope misfit $\operatorname{TFEM}(t, f)$, (b) time envelope misfit $\operatorname{TEM}(t)$, (c) and frequency envelope misfit $\operatorname{FEM}(f)$. (d) Time-frequency phase misfit TFPM $(t, f)$, (e) time phase misfit TPM $(t)$, (f) and frequency phase misfit FPM $(f)$. (i-g) Comparison of the laboratory (red) and numerical (blue) seismograms.
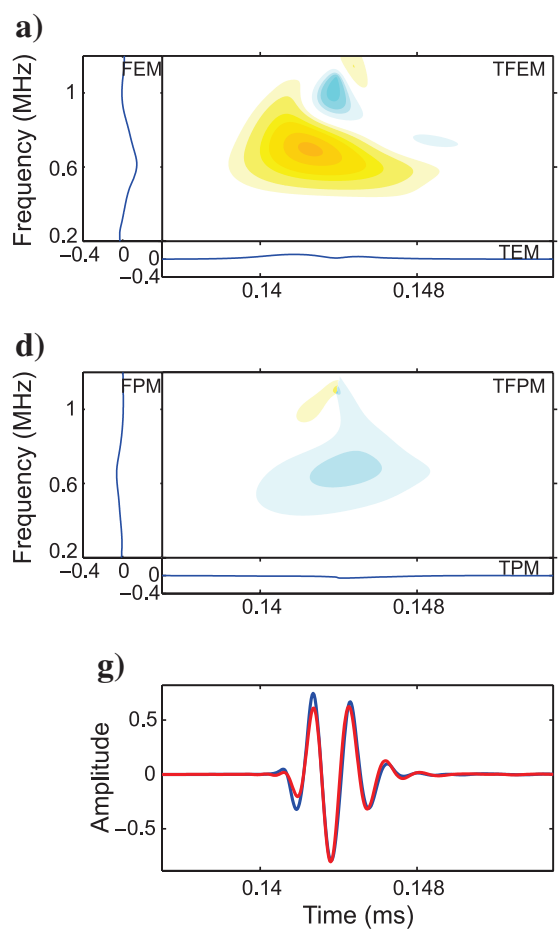

b)

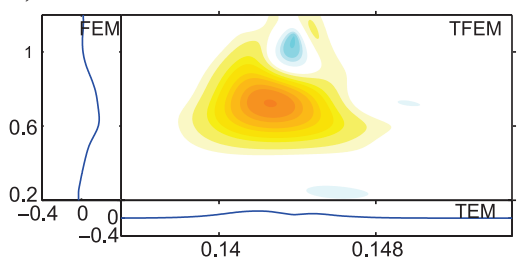

e)

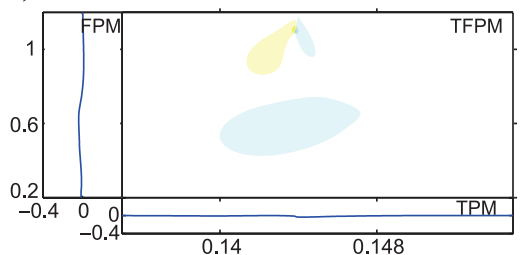

h)

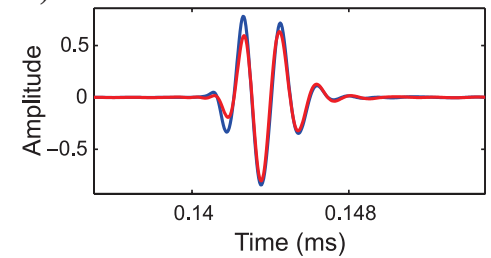

c)

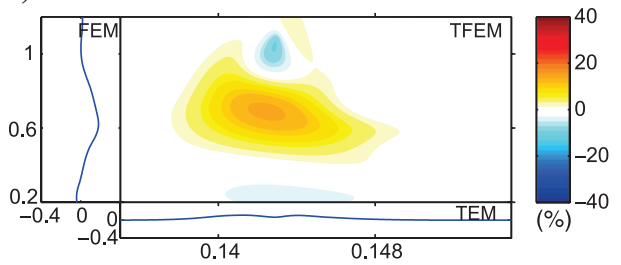

f)

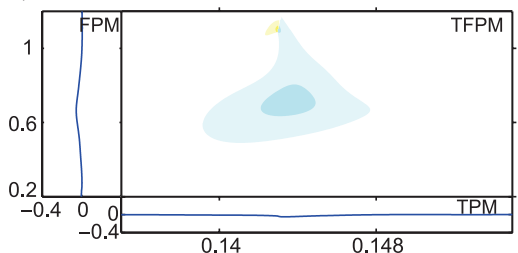

i)

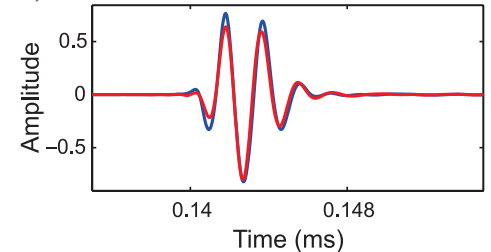

Figure 9. Time-frequency representation of the misfits for source positions NB1 (a), NB2 (b), and NB3 (c) for the narrow-beam transducer along the line Y200. (a) Time-frequency envelope misfit TFEM $(t, f)$, (b) time envelope misfit TEM $(t)$, (c) and frequency envelope misfit $\operatorname{FEM}(f)$. (d) Time-frequency phase misfit TFPM $(t, f)$, (e) time phase misfit TPM $(t)$, and (f) frequency phase misfit $\operatorname{FPM}(f)$. (g-i) Comparison of the laboratory (red) and numerical (blue) seismograms. 


$$
\mathrm{PM}=\sqrt{\frac{\sum_{f} \sum_{t}|\Delta P(t, f)|^{2}}{\sum_{f} \sum_{t}\left|W_{\mathrm{ref}}(f, t)\right|^{2}}} .
$$

The values of the normalized crosscorrelation coefficient, the rms misfit, the single-valued EM, and the single-valued PM for the selected traces are given in the Tables 1-4, respectively.

\section{Comparison for narrow-beam data}

We provide the more detailed comparison of three traces obtained using the numerical modeling and recorded in the laboratory for each of the three lines (see Figures 8-10). In these figures, we show the $\operatorname{TFEM}(t, f)$ and $\operatorname{TFPM}(t, f)$ for the three source positions along lines Y150, Y200, and Y250 together with the comparison of the laboratory and numerical traces in the time domain. Note that the range of the color scale and the misfit axes is the same for all plots and spans $\pm 40 \%$.

In Figure 8, the (a) trace corresponds to the reflection from the flat part of the model, the (b) trace to the reflection from the top of the truncated dome, and the (c) trace to the reflection from the flat part of the model in the vicinity of the cut of the dome. The qualitative comparison between $\operatorname{TFEM}(t, f)$ and $\operatorname{TFPM}(t, f)$ plots shows a good fit of the traces in terms of the shape and the phase of the signal, but it reveals discrepancies in terms of amplitude, which are quite low for reflections from the flat part of the model, but become significant over the top the dome.

Figures 9 and 10 present the same comparisons for lines Y200 and Y250, respectively. In Figure 9, all the traces correspond to the
Table 1. Normalized crosscorrelation coefficient.

\begin{tabular}{lccc}
\hline & Line Y150 & Line Y200 & Line Y250 \\
\hline NB1 & 0.9854 & 0.9816 & 0.9862 \\
NB2 & 0.9762 & 0.9884 & 0.9881 \\
NB3 & 0.9834 & 0.9850 & 0.9878 \\
BB1 & 0.9730 & 0.9816 & 0.9833 \\
BB2 & 0.9798 & 0.9611 & 0.9782 \\
BB3 & 0.9225 & 0.9467 & 0.9501 \\
\hline
\end{tabular}

Table 2. The rms misfit.

\begin{tabular}{lccc}
\hline & Line Y150 & Line Y200 & Line Y250 \\
\hline NB1 & 0.2417 & 0.2170 & 0.3875 \\
NB2 & 0.2792 & 0.2229 & 0.2364 \\
NB3 & 0.2981 & 0.2247 & 0.2180 \\
BB1 & 0.2461 & 0.2170 & 0.1995 \\
BB2 & 0.2490 & 0.2762 & 0.2279 \\
BB3 & 0.4287 & 0.3222 & 0.3271 \\
\hline
\end{tabular}

a)

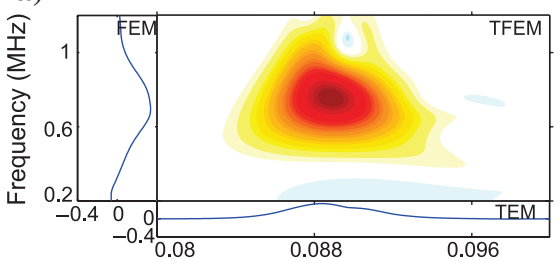

d)
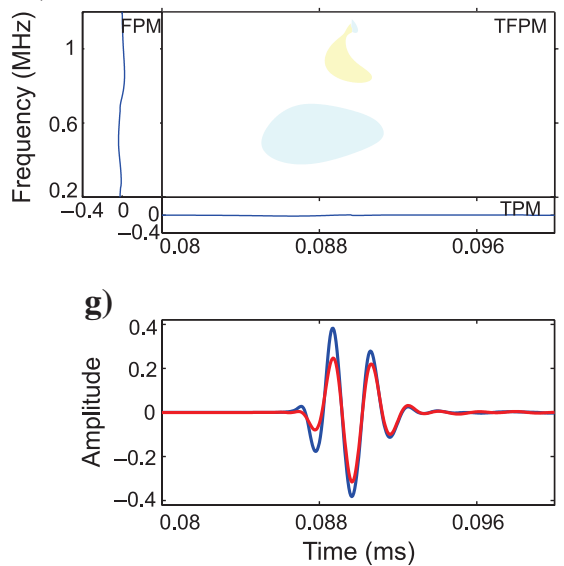

b)

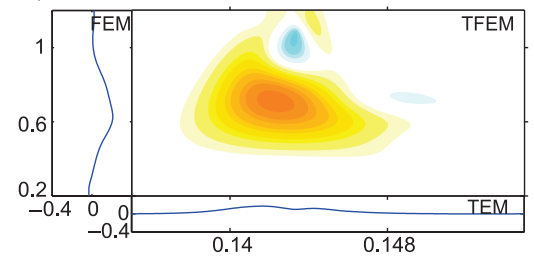

e)

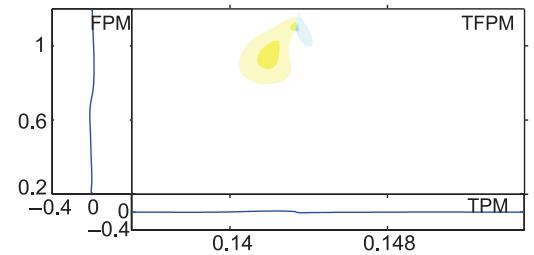

h)

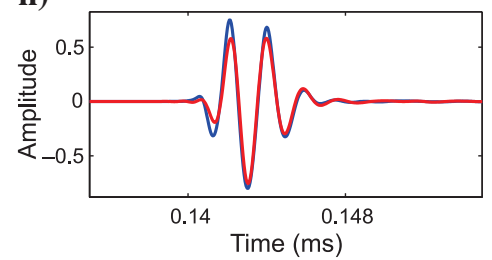

c)

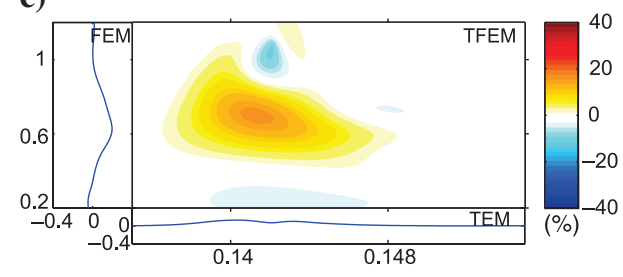

f)

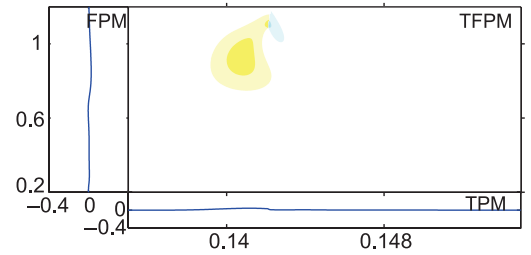

i)

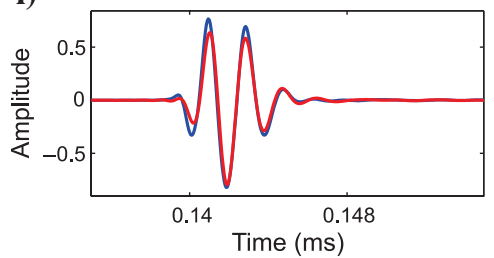

Figure 10. Time-frequency representation of the misfits for source positions NB1 (a), NB2 (b), and NB3 (c) for the narrow-beam transducer along the line Y250. (a) Time-frequency envelope misfit TFEM $(t, f)$, (b) time envelope misfit TEM $(t)$, (c) and frequency envelope misfit $\operatorname{FEM}(f)$. (d) Time-frequency phase misfits TFPM $(t, f)$, (e) time phase misfits TPM $(t)$, and (f) frequency phase misfits $\operatorname{FPM}(f)$. (g-i) Comparison of the laboratory (red) and numerical (blue) seismograms. 
reflection from the flat part of the model, and in Figure 10, the (a) trace corresponds to the reflection from the top of the full dome, and the (b) and (c) traces correspond to the reflection from the flat part of the model. Similar qualitative results, i.e., a good fit in terms of the shape and the phase of the signal, but discrepancies in terms of amplitude, can be observed for these two lines as well.

The qualitative results shown above are confirmed quantitatively by the high values of the normalized crosscorrelation coefficient (see Table 1, top) and the low values of PM (see Table 4, top) on the one hand, indicating that the phase fit is very good, and by relatively high values of the rms misfit and EM (see Tables 2 and 3 , top) for the reflections from the domes on the other hand. Note that the highest values are for the reflection from the top of the full dome. The problems with amplitude modeling over highly curved structures can be explained by oversimplification in description of reflection phenomenon with the PWRCs instead of the ERCs (see Ayzenberg et al., 2007) for more details on the topic). Using the PWRCs leads to increasing of relative errors in amplitude even for short offsets in the case of highly curved reflectors. The main source of error is that reflection of the incident wavefront from a convex spherical dome is numerically equivalent to reflection of an apparent wave with a higher front curvature at an apparent plane reflector. Note that the apparent front curvature is equal to the sum of the actual front curvature and the actual curvature of the reflector at the reflection point. Then, an apparent transducer is located at a twice as short distance from the reflector as the actual one is, which corresponds to the near-field region, and the ERCs should be applied. Therefore, the singly scattered wavefield modeled with the PWRCs contains larger amplitude errors.
Table 3. Single-valued EM.

\begin{tabular}{lccc}
\hline & Line Y150 & Line Y200 & Line Y250 \\
\hline NB1 & 0.1995 & 0.1267 & 0.3409 \\
NB2 & 0.2190 & 0.1752 & 0.1958 \\
NB3 & 0.2553 & 0.1561 & 0.1692 \\
BB1 & 0.1633 & 0.1945 & 0.1355 \\
BB2 & 0.1909 & 0.1422 & 0.1387 \\
BB3 & 0.1865 & 0.1794 & 0.1982 \\
\hline
\end{tabular}

Table 4. Single-valued PM.

\begin{tabular}{cccc}
\hline & Line Y150 & Line Y200 & Line Y250 \\
\hline NB1 & 0.0394 & 0.0545 & 0.0370 \\
NB2 & 0.0517 & 0.0394 & 0.0350 \\
NB3 & 0.0485 & 0.0487 & 0.0367 \\
BB1 & 0.0677 & 0.0562 & 0.0573 \\
BB2 & 0.0547 & 0.0785 & 0.0507 \\
BB3 & 0.1165 & 0.0955 & 0.0839 \\
\hline
\end{tabular}

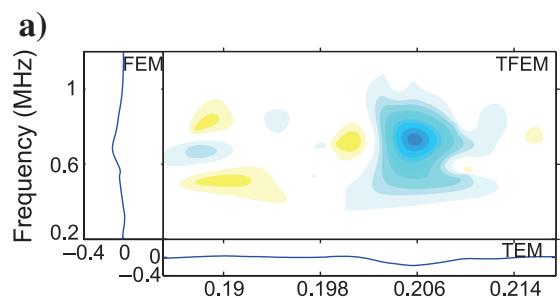

b)
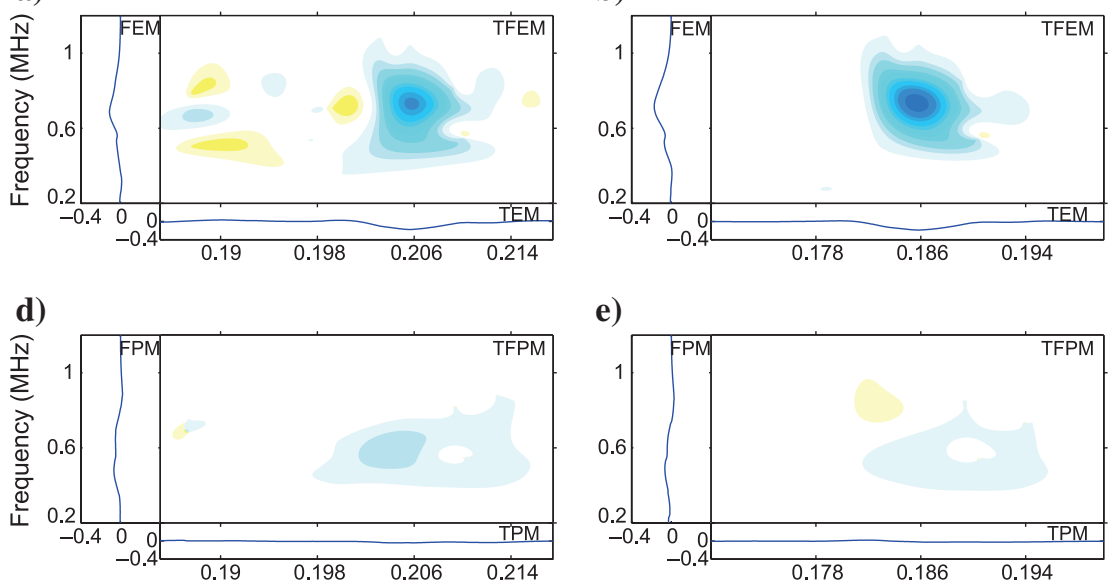

e)

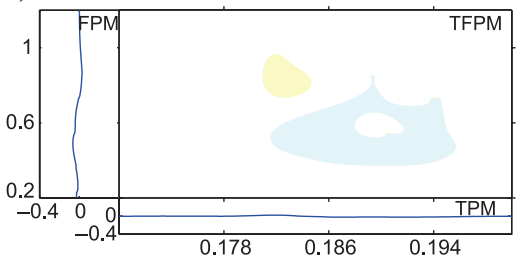

h)

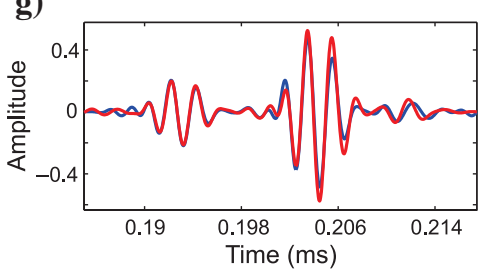

c)

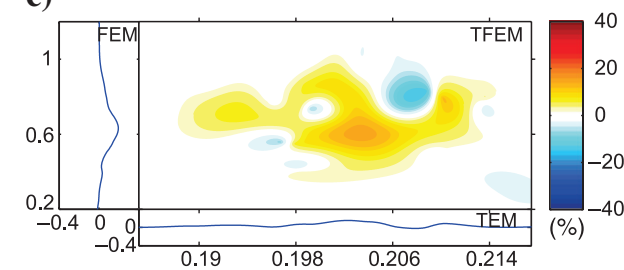

f)

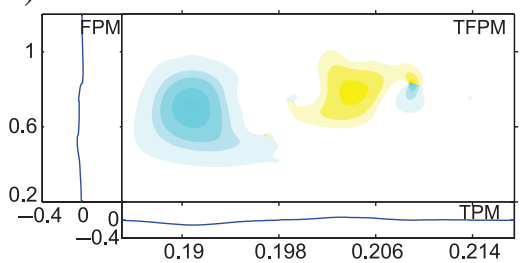

i)

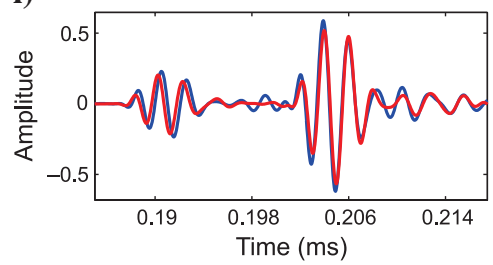

Figure 11. Time-frequency representation of the misfits for source positions BB1 (a), BB2 (b), and BB3 (c) for the broad-beam transducer along the line Y150. (a) Time-frequency envelope misfit $\operatorname{TFEM}(t, f)$, (b) time envelope misfit TEM $(t)$, and (c) frequency envelope misfit $\operatorname{FEM}(f)$. (d) Time-frequency phase misfit TFPM $(t, f)$, (e) time phase misfit TPM $(t)$, and (f) frequency phase misfit $\operatorname{FPM}(f)$. (g-i) Comparison of the laboratory (red) and numerical (blue) seismograms. 
a)

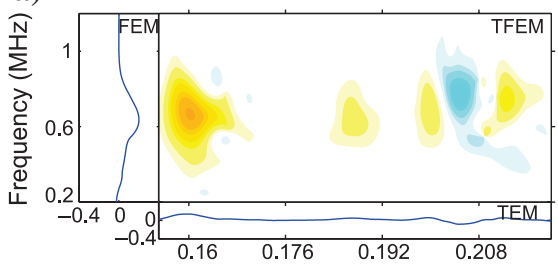

d)

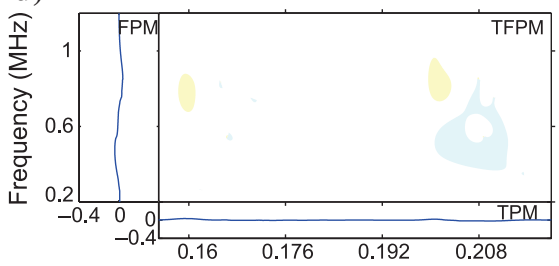

g)

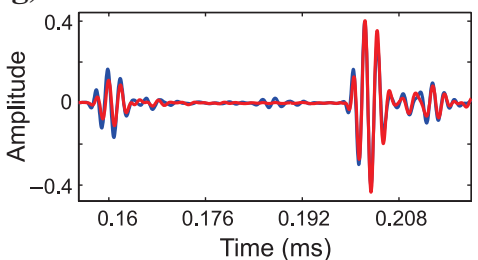

b)

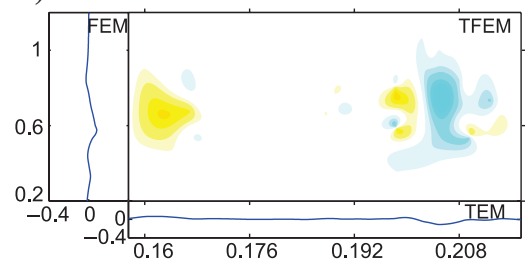

e)

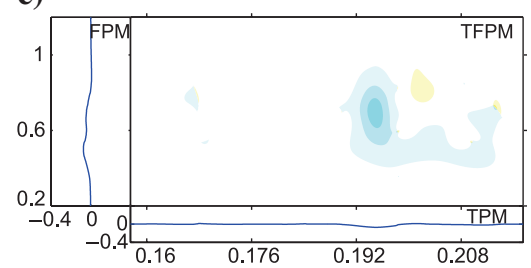

h)

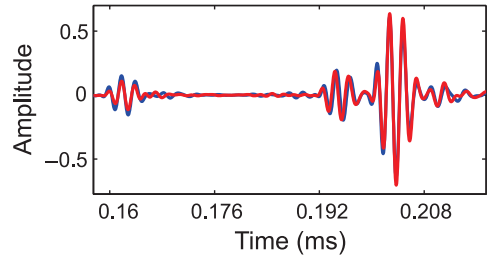

c)

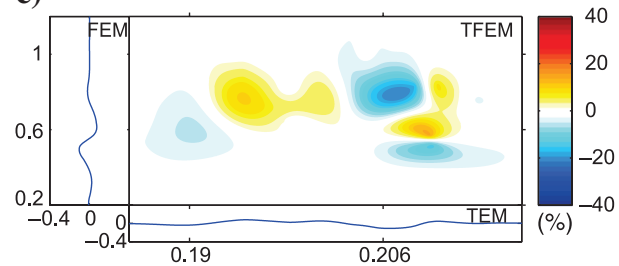

f)

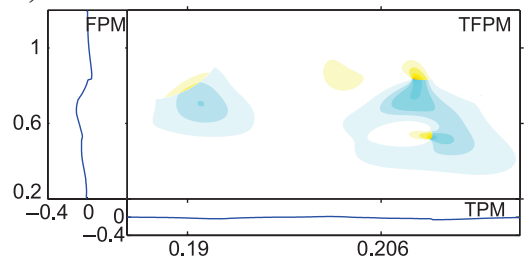

i)

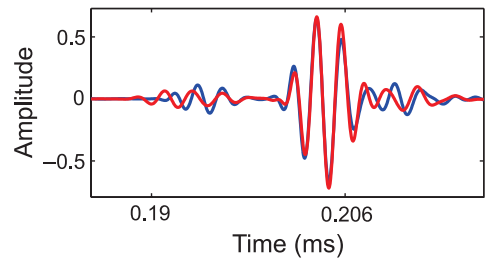

Figure 12. Time-frequency representation of the misfits for source positions BB1 (a), BB2 (b), and BB3 (c) for the broad-beam transducer along line Y200. (a) Time-frequency envelope misfit $\operatorname{TFEM}(t, f)$, (b) time envelope misfit TEM $(t)$, and (c) frequency envelope misfit $\operatorname{FEM}(f)$. (d) Time-frequency phase misfit TFPM $(t, f)$, (e) time phase misfit TPM $(t)$, and (f) frequency phase misfit FPM $(f)$. (g-i) Comparison of the laboratory (red) and numerical (blue) seismograms.

a)

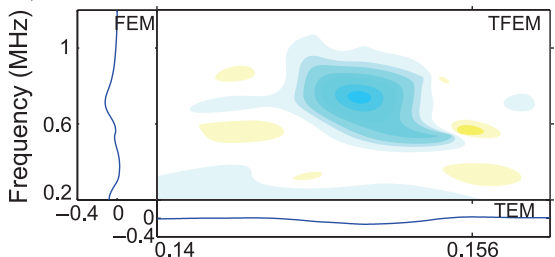

d)

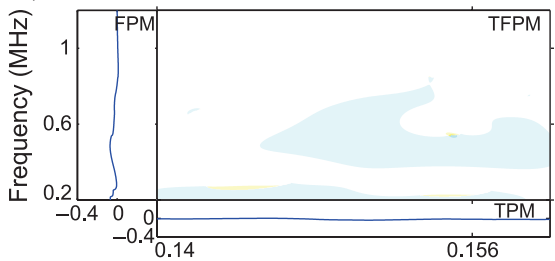

g)

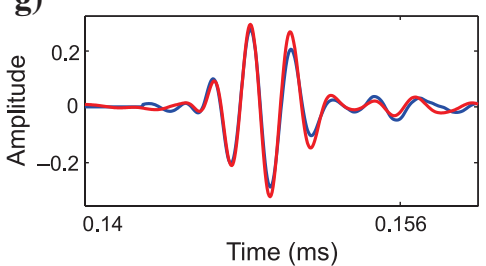

b)

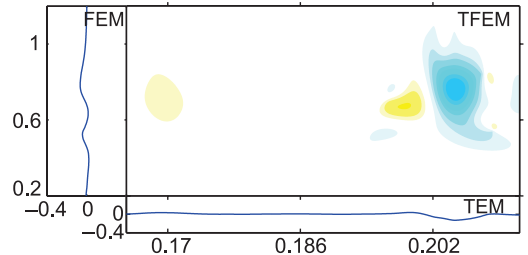

e)

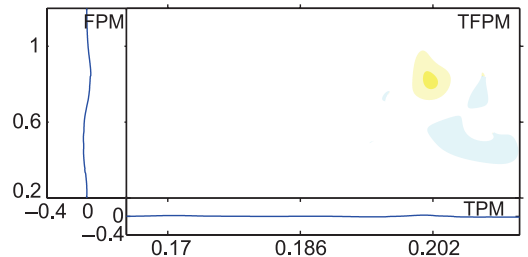

h)

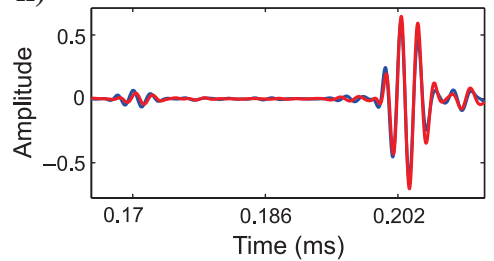

c)

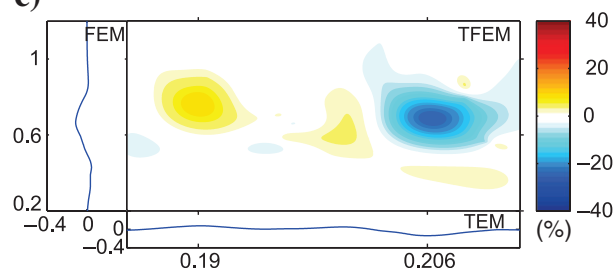

f)

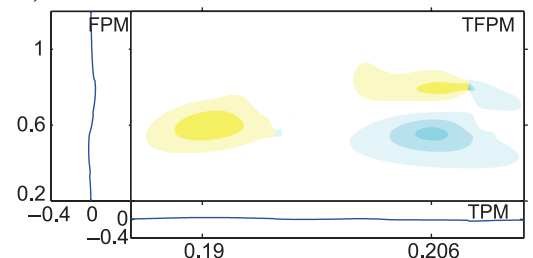

i)

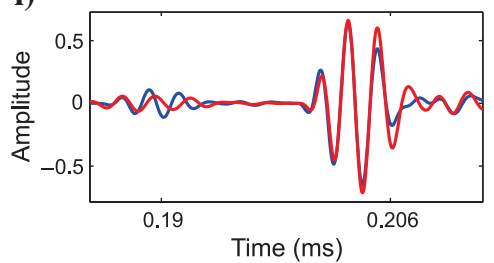

Figure 13. Time-frequency representation of the misfits for source positions BB1 (a), BB2 (b), and BB3 (c) for the broad-beam transducer along the line Y250. (a) Time-frequency envelope misfit $\operatorname{TFEM}(t, f)$, (b) time envelope misfit $\operatorname{TEM}(t)$, and (c) frequency envelope misfit $\operatorname{FEM}(f)$. (d) Time-frequency phase misfit TFPM $(t, f)$, (e) time phase misfit TPM $(t)$, and (f) frequency phase misfit $\operatorname{FPM}(f)$. (g-i) Comparison of the laboratory (red) and numerical (blue) seismograms. 


\section{Comparison for broad-beam data}

We provide a more detailed comparison of three traces obtained using numerical modeling and recorded in the laboratory for each of the three lines (see Figures 11-13). Note that the considered traces correspond to the same source positions as for the narrow-beam data. In these figures, we show the $\operatorname{TFEM}(t, f)$ and $\operatorname{TFPM}(t, f)$ for the three source positions along lines Y150, Y200, and Y250 together with the comparison of the laboratory and numerical traces in the time domain. The range of the color scale and the misfit axes is again the same for all plots and spans $\pm 40 \%$.

In Figure 11, the (a) trace corresponds to the reflection from the slope of the truncated dome and the flat part of the model, the (b) trace to the reflection from the top of the truncated dome, and the (c) trace to the diffraction from the cut of the truncated dome and the reflection from the flat part of the model. The qualitative comparison and the $\operatorname{TFEM}(t, f)$ and $\operatorname{TFPM}(t, f)$ plots show a good fit of the traces in terms of the shape and the amplitude of the signals, but it reveals a phase shift of the diffraction from the cut of the dome.

The qualitative result is confirmed quantitatively by the high values of the normalized crosscorrelation coefficient (see Table 1) and low values of the rms misfit, EM and PM (see Tables 2-4) for the first two traces, showing that the amplitude and the phase fit is very good. For the third trace, however, the visible phase shift of the diffraction results in a low crosscorrelation value and higher rms misfit and PM, while the EM is not affected. Larger time, amplitude, and phase errors for reflection from the cut of the dome can be explained by neglecting the double-scattering wavefield in the code. This event received by the transducer is created by the wedgelike structure from the vertical cut and horizontal part of reflector. It is composed of two sequential reflections from the cut and horizontal part and two sequential diffractions at the upper circular and lower straight edges having common tip points.

Figures 12 and 13 present the same comparisons for lines Y200 and Y250, respectively. In Figure 12, the (a) trace corresponds to the reflection from the slope of the out-of-plane full dome, the flat part of the model, and the slope of the out-of-plane truncated dome. The (b) trace corresponds to the reflection from the slope of the out-ofplane full dome and truncated dome and the flat part of the model. The (c) trace corresponds to the reflection from the slope of the fault and the reflection from the flat part of the model. In Figure 13, the (a) trace corresponds to the reflection from the top of the full dome, the (b) trace to the diffraction from the slope of the pyramid and the reflection from the flat part of the model, and the (c) trace to the reflection from the slope of the fault and the reflection from the flat part of the model. Again, qualitative comparison of the $\operatorname{TFEM}(t, f)$ and $\operatorname{TFPM}(t, f)$ plots shows a good fit of the traces in terms of the shape and the amplitude of the signals, except for the reflections from the slope of the fault, where small phase shifts can also be observed.

These results are confirmed quantitatively by slightly lower values of the normalized crosscorrelation coefficient (see Table 1, values for BB3) and higher values of rms misfit and PM (see Tables 2 and 4, values for BB3), while relatively low values of EM (see Table 3, values for BB3), showing that there are phase shifts, though the amplitude fit is good. It has been shown (Ayzenberg, 2008) that the time errors for reflected waves at plane reflectors and diffracted waves at straight edges modeled by the code do not exceed one sample. That is why the larger time shifts observed for the reflec- tions from the slope of the fault can be explained by a wrong tilt of the model used for modeling.

\section{CONCLUSIONS}

We have tested an alternative approach for benchmarking numerical methods for 3D wave propagation. This approach consists in comparing synthetic data computed using numerical modeling to laboratory data obtained for a known configuration. We have obtained the laboratory data by laboratory scale measurements of reflection of broadband pulses from a strong topographic environment immersed in water. The experiments have been performed in zero-offset seismic configuration using a physical model with strong 3D topographies and narrow- and broad-beam sources/ receivers. The features of the model have complicated surface curvatures, several edges, and vertices, which increase the diffraction effects produced on the wavefields significantly. These diffraction effects, together with the existence of shadow zones, make the laboratory experiments of interest. We have computed synthetic data by means of a discretized Kirchhoff integral method. The comparisons between synthetic and laboratory data exhibit a good quantitative fit in terms of time arrivals and acceptable fit in amplitudes for narrow-beam and broad-beam data sets. Errors in amplitude modeling can be explained by oversimplification in description of reflection phenomenon with the PWRCs.

\section{ACKNOWLEDGMENTS}

We would like to thank the INSIS Institute of the French CNRS, Aix-Marseille University, the Carnot Star Institute, the VISTA project, and the Norwegian Research Council through the ROSE project for financial support. We greatly acknowledge S. Devic (LMA Marseille) for curving the Marseille model. We thank the associate editor and the reviewers for constructive comments that helped to improve the paper.

\section{REFERENCES}

Aki, K., and P. G. Richards, 2002, Quantitative seismology: University Science Books.

Angona, F. A., 1960, Two-dimensional modeling and its application to seismic problems: Geophysics, 25, 468-482, doi: 10.1190/1.1438719.

Ayzenberg, M. A., 2008, Three-dimensional seismic diffraction modeling: Ph.D. thesis, Norwegian University of Science and Technology.

Ayzenberg, M. A., A. M. Aizenberg, H. B. Helle, K. D. Klem-Musatov, J. Pajchel, and B. Ursin, 2007, 3D diffraction modeling of singly scattered acoustic wavefields based on the combination of surface integral propagators and transmission operators: Geophysics, 72, no. 5, SM19-SM34, doi: 10.1190/1.2757616.

Ayzenberg, M. A., A. M. Aizenberg, H. B. Helle, K. D. Klem-Musatov, J. Pajchel, and B. Ursin, 2008, 3D modeling of acoustic Green's function in layered media with diffracting edges: 70th Annual International Conference and Exhibition, EAGE, Extended Abstracts, P052.

Ayzenberg, M. A., I. Tsvankin, A. M. Aizenberg, and B. Ursin, 2009, Effective reflection coefficients for curved interfaces in transversely isotropic media: Geophysics, 74, no. 5, WB33-WB53, doi: 10.1190/1.3197862.

Bilaniuk, N., and G. S. K. Wong, 1993, Speed of sound in pure water as a function of temperature: Journal of the Acoustical Society of America, 93 1609-1612, doi: 10.1121/1.406819.

Bleistein, N., 1984, Mathematical methods for wave phenomena: Academic Press.

Bretaudeau, F., D. Leparoux, O. Durand, and O. Abraham, 2011, Small-scale modeling of onshore seismic experiment: A tool to validate numerical modeling and seismic imaging methods: Geophysics, 76, no. 5 , T101-T112, doi: 10.1190/geo2010-0339.1. 
Campman, X. H., K. van Wijk, J. A. Scales, and G. C. Herman, 2005, Imaging and suppressing near-receiver scattered surface waves: Geophysics, 70, no. 2, V21-V29, doi: 10.1190/1.1884831.

Chapman, C. H., 2004, Fundamentals of seismic save propagation: Cambridge University Press.

Chen, H.-W., and G. A. McMechan, 1993, 3D physical modeling and pseudospectral simulation of seismic common-source data volumes: Geophysics, 58, 121-133, doi: 10.1190/1.1443341.

Cristini, P., N. Favretto-Cristini, A. Tantsereva, B. Ursin, A. M. Aizenberg, and D. Komatitsch, 2012, Laboratory benchmarks vs. synthetic modeling of seismic wave propagation in complex environments (BENCHIE project): Results for a spectral-element method and the tip-wave superposition method: Proceedings of Meetings on Acoustics, 17, 070024.

Ebrom, D. A., and J. A. McDonald, 1994, Seismic physical modeling: SEG.

Ekanem, A. M., J. Wei, X.-Y. Li, M. Chapman, and I. G. Main, 2013, Pwave attenuation anisotropy in fractured media: A seismic physical modeling study: Geophysical Prospecting, 61, 420-433, doi: 10.1111/j.13652478.2012.01127.x.

Fehler, M., and P. J. Keliher, 2011, SEAM Phase I: Challenge of subsalt imaging in Tertiary basins, with emphasis on deepwater Gulf of Mexico: SEG.

French, W. S., 1974, Two-dimensional and three-dimensional migration of model-experiment reflection profiles: Geophysics, 39, 265-277, doi: 10 $.1190 / 1.1440426$

Futterman, W. I., 1962, Dispersive body waves: Journal of Geophysical Research, 67, 5279-5291, doi: 10.1029/JZ067i013p05279.

Grannemann, W. W., 1956, Diffraction of a longitudinal pulse from a wedge in a solid: Journal of the Acoustical Society of America, 28, 494-497, doi: $10.1121 / 1.1908368$.

Haddon, R. A. W., and P. W. Buchen, 1981, Use of Kirchhoff's formula for body wave calculations in the earth: Geophysical Journal of the Royal Astronomical Society, 67, 587-598, doi: 10.1111/j.1365-246X.1981 .tb06939.x.

Harris, G. R., 1981, Review of transient field theory for a baffled planar piston: Journal of the Acoustical Society of America, 70, 10-20, doi: $10.1121 / 1.386687$

Hilterman, F., 1970, Three-dimensional seismic modeling: Geophysics, 35, 1020-1037, doi: $10.1190 / 1.1440140$.

Houbiers, M., E. Wiarda, J. Mispel, D. Nikolenko, D. Vigh, B. E. Knudsen, M. Thompson, and D. Hill, 2012, 3D full-waveform inversion at Mariner: A shallow North Sea reservoir: 82nd Annual International Meeting, SEG, Expanded Abstracts, doi: 10.1190/segam2012-0242.1.

Howes, E., L. Tejada-Flores, and L. Randolph, 1953, Seismic model study: Journal of the Acoustical Society of America, 25, 915-921, doi: 10.1121/ 1.1907218 .

Howson, C. D., and M. C. Sinha, 1984, A comparison of ultrasonic and synthetic seismograms for a laterally varying structure: Geophysical Journal of the Royal Astronomical Society, 77, 517-529, doi: 10.1111/j.1365246X.1984.tb01946.x.

Kolsky, H., 1956, The propagation of stress pulses in viscoelastic solids: Philosophical Magazine, 1, 693-710, doi: 10.1080/14786435608238144.

Kristeková, M., J. Kristek, and P. Moczo, 2009, Time-frequency misfit and goodness-of-fit criteria for quantitative comparison of time signals: Geophysical Journal International, 178, 813-825, doi: 10.1111/j.1365246X.2009.04177x

Lines, L., K. Innanen, F. Vasheghani, and J. Wong, 2012, Experimental confirmation of "reflections on Q": 82nd Annual International Meeting, SEG, Expanded Abstracts, doi: 10.1190/segam2012-0187.1.

Lo, T.-W., M. N. Toksöz, S.-H. Xu, and R.-S. Wu, 1988, Ultrasonic laboratory tests of geophysical tomographic reconstruction: Geophysics, 53, 947-956, doi: 10.1190/1.1442531.

Macdonald, C., P. M. Davis, and D. D. Jackson, 1987, Inversion of reflection traveltimes and amplitudes: Geophysics, 52, 606-617, doi: 10.1190/1 .1442330 .
Moczo, P., J.-P. Ampuero, J. Kristek, S. M. Day, M. Kristeková, P. Pazak, M. Galis, and H. Igel, 2006, Comparison of numerical methods for seismic wave propagation and source dynamics - The SPICE code validation: Presented at International Symposium on the Effects of Surface Geology on Seismic Motion.

Oliver, J., F. Press, and M. Ewing, 1954, Two-dimensional model seismology: Geophysics, 19, 202-219, doi: 10.1190/1.1437982.

Pant, D. R., S. A. Greenhalgh, and B. Zhou, 1992, Physical and numerical model study of diffraction effects on seismic profiles over simple structures: Geophysical Journal International, 108, 906-916, doi: 10.1111/j 1365-246X.1992.tb03479.x.

Pratt, R., 1999, Seismic waveform inversion in the frequency domain. Part I: Theory and verification in a physical scale model: Geophysics, 64, 888901, doi: 10.1190/1.1444597.

Rieber, F., 1936, Visual presentation of elastic wave patterns under various structural conditions: Geophysics, 1, 196-218, doi: 10.1190/1.1437093.

Robertsson, J. O. A., B. Bednar, J. Blanch, C. Kostov, and D. J. van Manen, 2007 , Introduction to the supplement on seismic modeling with applications to acquisition, processing, and interpretation: Geophysics, 72, no. 5 , SM1-SM4, doi: 10.1190/1.2755959.

Roever, W. L., T. F. Vining, and E. Strick, 1959, Propagation of elastic wave motion from an impulsive source along a fluid/solid interface: Philosophical Transactions of the Royal Society, 251, 455-523, doi: 10.1098/rsta .1959 .0009

Sherlock, D. H., and B. J. Evans, 2001, The development of seismic reflection sandbox modeling: AAPG Bulletin, 85, 1645-1659, doi: 10.1306/ 8626CCE9-173B-11D7-8645000102C1865D.

Sherlock, D. H., J. McKenna, and B. J. Evans, 2000, Time-lapse 3D seismic physical modeling: Exploration Geophysics, 31, 310-314, doi: 10.1071/ EG00310.

Sivaji, C., O. Nishizawa, G. Kitagawa, and J. Fukushima, 2002, A physical model study of the statistics of seismic waveform fluctuations in random heterogeneous media: Geophysical Journal International, 148, 575-595, doi: 10.1046/j.1365-246x.2002.01606.x.

Stewart, R. R., N. Dyaur, B. Omoboya, J. J. S. de Figueiredo, M. Willis, and S. Sil, 2013, Physical modeling of anisotropic domains: Ultrasonic imaging of laser-etched fractures in glass: Geophysics, 78, no. 1, D11-D19, doi: 10.1190/geo2012-0075.1.

Tantsereva, A., B. Ursin, N. Favretto-Cristini, P. Cristini, D. Komatitsch, and A. M. Aizenberg, 2012, Comparison of numerical seismic modeling results with acoustic water-tank data: 74th Annual International Conference and Exhibition, EAGE, Extended Abstracts, P357.

Tygel, M., and B. Ursin, 1999, Weak-contrast edge and vertex diffractions in anisotropic elastic media: Wave Motion, 29, 363-373, doi: 10.1016/ S0165-2125(98)00044-4.

Ursin, B., 2004, Parameter inversion and angle migration in anisotropic elastic media: Geophysics, 69, 1125-1142, doi: 10.1190/1.1801931.

Virieux, J., H. Calandra, and R.-E. Plessix, 2011, A review of the spectral, pseudo-spectral, finite-difference and finite-element modelling techniques for geophysical imaging: Geophysical Prospecting, 59, 794-813, doi: 10 $.1111 / \mathrm{j} .1365-2478.2011 .00967 . x$.

Virieux, J., and S. Operto, 2009, An overview of full-waveform inversion in exploration geophysics: Geophysics, 74, no. 6, WCC1-WCC26, doi: 10 $1190 / 1.3238367$

Wilks, D. S., 2011, Statistical methods in the atmospheric sciences, 3rd ed.: Academic Press.

Woods, J. P., 1956, Composition of reflections: Geophysics, 21, 261-276, doi: $10.1190 / 1.1438223$

Zemanek, J., 1971, Beam behavior within the near-field of a vibrating piston: Journal of the Acoustical Society of America, 49, 181-191, doi: 10 $.1121 / 1.1912316$

Zhang, F., C. Juhlin, and M. Ivandic, 2012, Application of seismic waveform inversion for time-lapse monitoring of $\mathrm{CO}_{2}$ injection: A real data example from Ketzin, Germany: 82nd Annual International Meeting, SEG, Expanded Abstracts, doi: 10.1190/segam2012-0684.1. 